\title{
Lower-hybrid (LH) oscillitons evolved from ion-acoustic (IA)/ ion-cyclotron (IC) solitary waves: effect of electron inertia
}

\author{
J. Z. G. $\mathrm{Ma}^{1}$ and A. Hirose ${ }^{2}$ \\ ${ }^{1}$ Space Science Branch, Canadian Space Agency, 6767, Route De I'Aéroport, Saint-Hubert, Québec, J3Y 8Y9, Canada \\ ${ }^{2}$ Department of Physics and Engineering Physics, University of Saskatchewan, 116 Science Place, Saskatoon, Saskatchewan, \\ S7N 5E2, Canada
}

Received: 15 January 2010 - Revised: 21 April 2010 - Accepted: 26 April 2010 - Published: 7 May 2010

\begin{abstract}
Lower-hybrid (LH) oscillitons reveal one aspect of geocomplexities. They have been observed by rockets and satellites in various regions in geospace. They are extraordinary solitary waves the envelop of which has a relatively longer period, while the amplitude is modulated violently by embedded oscillations of much shorter periods. We employ a two-fluid (electron-ion) slab model in a Cartesian geometry to expose the excitation of LH oscillitons. Relying on a set of self-similar equations, we first produce, as a reference, the well-known three shapes (sinusoidal, sawtooth, and spiky or bipolar) of parallel-propagating ion-acoustic (IA) solitary structures in the absence of electron inertia, along with their Fast Fourier Transform (FFT) power spectra. The study is then expanded to illustrate distorted structures of the IA modes by taking into account all the three components of variables. In this case, the ion-cyclotron (IC) mode comes into play. Furthermore, the electron inertia is incorporated in the equations. It is found that the inertia modulates the coupled IA/IC envelops to produce LH oscillitons. The newly excited structures are characterized by a normal low-frequency IC solitary envelop embedded by highfrequency, small-amplitude LH oscillations which are superimposed upon by higher-frequency but smaller-amplitude IA ingredients. The oscillitons are shown to be sensitive to several input parameters (e.g., the Mach number, the electronion mass/temperature ratios, and the electron thermal speed). Interestingly, whenever a LH oscilliton is triggered, there occurs a density cavity the depth of which can reach up to $20 \%$ of the background density, along with density humps on both sides of the cavity. Unexpectedly, a mode at much lower frequencies is also found beyond the IC band. Future studies are
\end{abstract}

Correspondence to: J. Z. G. Ma

(johnzg.ma@asc-csa.gc.ca) finally highlighted. The appendices give a general dispersion relation and specific ones of linear modes relevant to all the nonlinear modes encountered in the text.

\section{Introduction}

Nonlinear waves have increasingly drawn much attention in the study of geocomplexities in last decades. They are ubiquitous in space and laboratories under different plasma conditions. Since the first soliton (also called a solitary wave) was noticed by John Scott Russell in 1834, the first equation (i.e., the Korteweg-de Vries, $\mathrm{KdV}$, equation) was derived to describe weakly dispersive, nonlinear water waves in 1895 , and the first prediction was made about the existence of the non-wave structures in plasmas (called the Bernstein-GreenKruskal, BGK, mode) in 1957, many branches have been developed to meet the needs of solving different problems relevant to the construction, maintenance, propagation, and effects of solitary structures.

A focus is on large-scale, finite-amplitude, solitary envelops. The study can be traced far back to 1970 s when Shukla and Yu (1978) offered exact stationary solutions for ion-acoustic (IA) solitons propagating obliquely in a twocomponent (electron and ion), low- $\beta$, non-isothermal plasma system, with an assumption that ions do not have a polarization drift in a constant magnetic field. In this case, the electron inertia was neglected due to the much smaller mass than that of ions. Later, the limit of the polarization drift was relaxed by Yu et al. (1980) and a generalized result was obtained. Meanwhile, Temerin et al. (1979) found three shapes of electrostatic solitary waves: sinusoidal, sawtooth, and spiky/bipolar. In a generalized study, Lee and Kan (1981) obtained nonlinear IA and ion-cyclotron (IC) waves (the authors also mentioned another type, so called "IA solitons"; however, by reproducing the results, we easily confirmed that

Published by Copernicus Publications on behalf of the European Geosciences Union and the American Geophysical Union. 
it is not an independent type but the IA solitary waves with a longer period). Nakamura and Sugai (1996) studied nonlinear waves in a three-component (warm ion, cold and energetic electrons), unmagnetized plasma system. They pointed out that a pseudo-potential method (i.e., the "Sagdeev potential") is more suitable than the KdV approach to produce results applicable to experiments. In such a system, Chatterjee and Roychoudhury (1997) found that, when the ion temperature increases, the amplitude of the IA solitary waves increases when the corresponding self-similar position (denoted by $\xi=x-V t$, where $x$ is the 1-D coordinate, $V$ is the phase speed of solitons, and $t$ is the time) shifts to the origin. For a system containing multi-ionic components but ions are cold, Das et al. (2000) claimed that a power expansion technique leads to higher order nonlinear IA wave equations; these equations yield various solitary waves (such as, spiky solitons, double layers, etc.) depending on plasma parameters.

More important, Jovanović and Shukla (2000) found a fully nonlinear coherent solution for solitary structures (called "electron-holes" in the text) in the magnetosphere. They took into account a low-frequency (LF) ion dynamics, and exposed a typical cylindrically-symmetric hole of a Larmor-radius size. The results were used to explain FAST/POLAR observations of antisymmetric bipolar pulses in the parallel direction to localized magnetic field lines and unipolar ones in the perpendicular plane. In addition, Mamun et al. (2002) employed the power expansion technique to study the properties of obliquely propagating electronacoustic (EA) solitary waves in a magnetized plasma system of three components: a cold magnetized electron fluid, hot electrons obeying a non-isothermal vortex-like distribution, and stationary ions. It was found that the external magnetic field and the obliqueness of the solitary waves could greatly change the amplitude and the width of solitons, and, positive potential solitons correspond to cold electron density holes/cavitons (note that these electron holes are "ion clumps" as defined by Dupree, 1972; and naturally, the potential is positive). Moreover, Reddy et al. (2002) considered a two-component (cold ions and warm electrons), homogeneous, magnetized system. The authors verified that parallel-propagating solitary waves have structures of sinusoidal, sawtooth, and highly spiky waveforms, and, the highly spiky waveforms have periods ranging from IC to IA frequencies. Likewise, Bharuthram et al. (2002) considered a homogeneous magnetized plasma system consisting of Boltzmann electrons and warm ions, aiming at the nonlinear solitary structures arising from a coupling between the IA and IC waves. The authors not only obtained the wellknown three waveforms, but also suggested that a finite ion temperature suppresses the IC nonlinearity and enhances the IA nonlinearity. Furthermore, in order to explain the fine structures in the auroral kilometric radiation, Pottelette et al. (2003) studied the excitation of IA solitons (called "electrostatic shocks" in the text) in a unmagnetized ion-electron system. They used the Sagdeev potential to derive the amplitude, speed, and width of the localized IA shocks in the fluid approximation. Such electrostatic nonlinear structures were considered to be necessary for auroral electron acceleration up to the observed energies of $\sim 10 \mathrm{keV}$. Recently, Ma and Hirose (2009a) performed a parameterized study on IA solitary waves by employing the Sagdeev potential in a magnetized plasma system consisting of warm ions, background electrons, and energetic electrons.

It deserves to mention some other topics in the study of electrostatic solitary waves, so as to show the robust growth of this subject in space physics. One of the subdivisions is for small-scale plasma systems. In these systems, influences brought about by either the finite radius of a flux tube (Gradov et al., 1984,1985), or the boundary in slab models (Vladimirov et al., 1993) cannot be neglected anymore. Luckily, as indicated by these authors, the effects are generally limited in the boundary layers and do not have an impact on bulk solitary wave propagations. Another branch is the study of EA solitary waves. These type of waves are known to contribute most of higher-frequency electrostatic noises than IC/IA waves. Different from the IC case where the electron inertia is neglected, the study assumes motionless ions. The first study was done by Dubouloz (1993), followed by e.g., Mamun et al. (2002), Berthomier et al. (2000, 2003), Shukla et al. (2004), Singh and Lakhina (2004), Kakad et al. (2007), Lakhina et al. (2008). Specially, Pottelette and Berthomier (2009) set up a model which is useful to explain observations. The third fork lies in the study on the effects of the centrifugal and Coriolis forces on the propagation of solitary waves. The work was initiated by Stenflo (1990), and followed by Yu and Stenflo (1991), Stenflo and Yu (1992, 1995), Shi et al. (2001, 2005, 2008) and Ma (2010).

The packets mentioned above constitute simple nonlinear waves; i.e., the Fast Fourier Transform (FFT) power spectra of the envelops are featured by a single frequency only, as well as its harmonics. To our surprise, observations of high-sensitivity rockets and satellites in last 20 years exposed a new type of more complicated solitary waves. They are associated with the lower-hybrid (LH) mode, accompanied by density depletions. The MARIE sounding rocket made the first definitive observations of the LH cavities (LaBelle et al., 1986), and the waveforms have been exhibited by many implemented space projects, such as, the FREJA satellite (Dovner et al., 1994; Eriksson et al., 1994; Pécseli et al., 1996; Høymork et al., 2001), the Alaska-93 sounding rocket (Delory, 1996), the AMICIST sounding rocket (Pinçn et al., 1997), the Polar satellite (Cattell et al., 1998, 1999), the FAST satellite (McFadden et al., 1998; Pottelette et al., 1999), the GEODESIC sounding rocket (Knudsen et al., 2003; Burchill et al., 2004), and the Viking and Cluster satellites (Tjulin et al., 2003, 2004). Schuck et al. (2003) made a detailed review on related observations and simulations. 
The new packets exhibit violent modulations in amplitudes of relatively slowly-varying, classical solitary structures, performed by embedded quickly-varying, small-amplitude oscillations. Such a structure was called "oscillitons" (Sauer et al., 2001), but there, and in subsequent papers by the same group of authors (e.g., Sauer et al., 2002, 2003; Dubinin et al., 2002, 2003a, b, c, 2007; McKenzie et al., 2004; Cattaert and Verheest, 2005; Sydora et al., 2007), it was used in the sense of nonlinear electromagnetic "whistler" structures, which can exist at parallel and supposedly also at oblique propagation with respect to the static, background magnetic field (F. Verheest, personal communication, 2010). The modulation was present in either a multi-species system (more than two types of charged particles) responsible for observed IC oscillitons (e.g., Sauer et al., 2001, 2003), or an electronion system to explain coherent whistler waves and oscillitons (e.g., Cattaert and Verheest, 2005; Sydora et al., 2007). Notice that in this article and following ones, we expand the intrinsic meaning of the terminology and use it to describe amplitude-modulated solitary packets, either the waves are excited electrostatically or electromagnetically, and either in a non-dusty plasma system or a dusty one. Figure 1 gives an example to show the observed features of "LH-oscilliton" structures, adapted from Fig. 3 of Cattell et al. (1998). The measurement was performed by the Polar satellite at the plasma sheet boundary. The large amplitudes of $E_{\mathrm{x}}$ and $E_{\mathrm{y}}$ can be up to $40 \mathrm{mV} / \mathrm{m}$. Each of the LH packets after 02:05:25.7 lasts $\lesssim 0.1 \mathrm{~s}$ (about 3 ion gyroperiods) appearing in density cavities. The bursty nature exhibits the modulation of the LH mode (see details in Cattell et al., 1998).

That LH solitary structures are of great importance in the study of nonlinear processes is by virtue of the fact that they are the prime candidate for the acceleration of ions and generation of "ion conics" in the high-latitude ionosphere and magnetosphere (see comprehensive contributions in 198090s by, e.g., Chang and Coppi, 1981; Retterer et al., 1986; Kintner et al., 1992; Chang, 1993, and references therein). However, an effective modulational mechanism of "oscillitons" had not been proposed till Kourakis and Shukla (2005). The authors provided a complicated methodological formulation to suggest that the modulation may be due to parametric interactions between different modes or, simply, to the nonlinear (self-)interaction of some wave itself.

We checked observations after investigating the LH instabilities (Ma and Hirose, 2009b). We found that the modulation may be easily understood by noticing the role played by the electron inertia. For example, the FAST satellite showed the modulations of electrons in the excitation of solitary waves (McFadden et al., 1998): in an auroral density cavity containing ion beams, electrons inside an ion-beam region are modulated at hydrogen cyclotron frequency $208 \mathrm{~Hz}$, while the ones outside it are at $\sim 120 \mathrm{~Hz}$, along with an identified LH frequency at $\sim 450 \mathrm{~Hz}$ which was found to merge to IC waves continuously at the boundary of the ion-beam region. This indicates that the role played by the electron

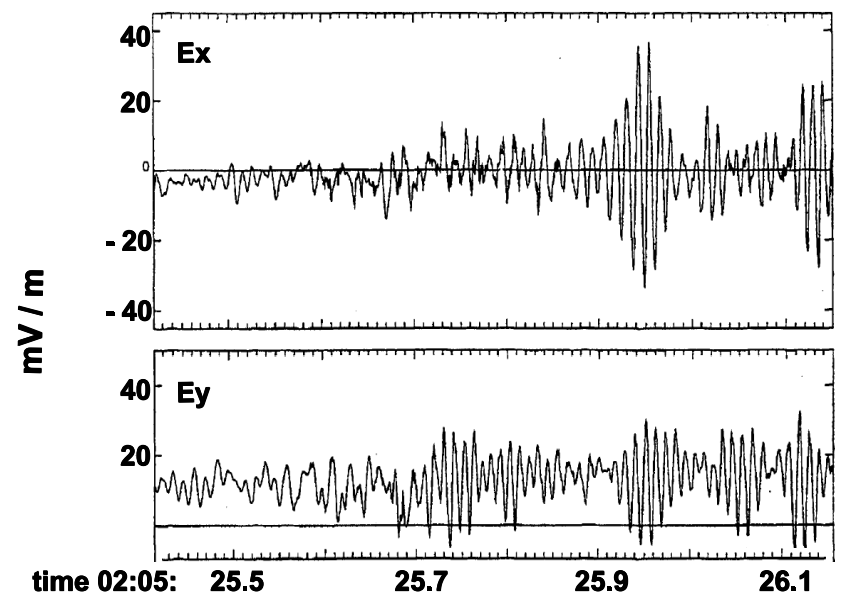

Fig. 1. Nonlinear "oscilliton" structures associated with the LH mode (adapted from Fig. 3 of Cattell et al., 1998). The measurement was performed by the Polar satellite at the plasma sheet boundary. The bursty nature after 02:05:25.7 exhibits the modulation of the LH mode, see, e.g., a detailed discussion by Cattell et al. (1998).

inertia becomes dominant outside the ion-beam region. Besides, the Cluster satellites exposed that the widths of the cavities lie between the ion gyroradius $r_{\mathrm{i}}=v_{\mathrm{T}_{\mathrm{i}}} / \Omega_{\mathrm{i}}$ (where $v_{\mathrm{T}_{\mathrm{i}}}$ is the ion thermal speed and $\Omega_{\mathrm{i}}$ is the ion gyro-frequency) and the electron inertial length $r_{\mathrm{e}}=c / \omega_{\text {pe }}$ (where $c$ and $\omega_{\text {pe }}$ are the speed of light and electron plasma frequency, respectively) (Tjulin et al., 2004). This reveals that the electron inertia is an important parameter due to the fact that observed LH phenomena are connected, more or less, to inertial Alfvén waves (Shapiro, 1998), the nonlinear mode of which was found to modulate spatially the electron density and energy (Knudsen, 1996).

What is more, in cases where the electron inertia is neglected, in the linear regime, IA/IC modes are excited (e.g., Boyd and Sanderson, 2003); while in the nonlinear regime, it is the IA/IC solitary waves, rather than LH ones, that are initiated with either small amplitudes or large amplitudes, where electrons are free to response to ion kinetics, satisfying the charge-nuetrality condition (e.g., Reddy et al., 2002). On the contrary, if the electron inertia is present, plasma particles are coupled with each other which prevents electrons from becoming unbounded. In the linear regime, high-frequency $\left(\omega \geq \Omega_{\mathrm{i}}\right)$ electrostatic LH instabilities are excited (e.g., Hirose and Alexeff, 1972). By contrast, in the nonlinear regime, on one hand, the inertia constrains small-amplitude IA solitary waves (Kuehl and Zhang, 1991); on the other hand, for largeamplitude ones, the inertia enhances the IC amplitudes (Sen et al., 2008).

As a matter of fact, the effects of electron inertia have already been discussed extensively in other fields, such as, linear tearing mode and nonlinear magnetic islands (Shivamoggi, 1997), magnetic reconnection (Al-Salti and Shivamoggi, 2003), gyrokinetic turbulence (Jones and Parker, 
2003), acoustic instabilities (Merlino and D'Angelo, 2005), and Alfvén compressional wave of gravitational instabilities (Uberoi, 2009). Manifestly, there should exists an intrinsic interplay via the electron inertia between IA/IC solitary structures and "LH oscillitons". Motivated by Kourakis and Shukla (2005)'s work which provides a generic methodology applicable to a variety of electrostatic modes, we aim at finding a formulation to explain the influence of the electron inertia on the transition from IA/IC solitary waves to LH oscillitons.

Enlightened by Sauer et al. (2003)'s work, we make use of a fluid description. In order to concentrate on the mechanism of the modulation played by the electron inertia, and gain important insights into more complicated situations (such as a multi-species system), while still being able to illustrate our approach clearly, we consider a slab model, as described in Sect. 2, to formulate a collision-free, two-fluid (electronion) system in a Cartesian geometry. We start from introducing basic parallel-propagating IA solitary waves in Sect. 3, where the electron inertia is not taken into consideration, and the FFT spectra are shown. Then, in Sect. 4, we describe distorted IA/IC solitary waves. On the basis of these studies, we investigate in Sect. 5 the impact of the electron inertia on the possible excitation and propagation of nonlinear $\mathrm{LH}$ oscillitons by a parameterized study through a few input parameters. A simulation is performed. Finally, in Sect. 6, we summarize the results and have some discussions. The Appendices give generalized and specific dispersion relations of linear modes, corresponding to all the nonlinear ones discussed in the text.

\section{Nonlinear, two-fluid equations: a slab model}

We consider that an external magnetic field permeates through regions visited by satellites (e.g., auroral acceleration regions by FAST, bow shock and magnetopause by Cluster). It has three components:

$\boldsymbol{B}_{\mathrm{ext}}=B_{\mathrm{x} 0} \hat{\boldsymbol{e}}_{\mathrm{x}}+B_{y 0} \hat{\boldsymbol{e}}_{\mathrm{y}}+B_{\mathrm{z} 0} \hat{\boldsymbol{e}}_{\mathrm{z}}$

in a Cartesian geometry where $B_{j 0}(j=\mathrm{x}, \mathrm{y}, \mathrm{z})$ is constant in the direction of $j$ along which $\hat{\boldsymbol{e}}_{j}$ is the unit vector. In these regions, the plasma pressure is much smaller than the magnetic pressure, i.e., $\beta \ll 1$. Plasma waves excited are thus electrostatic in general, rather than electromagnetic, with $k_{\mathrm{z}} \ll k_{\perp}$ due to the parallel magnetization of particles (where $k_{\mathrm{z}}$ and $k_{\perp}$ denote parallel and transverse wavenumbers, respectively). The Landau damping can be neglected due to the fact that the phase speed $v_{\mathrm{p}}$ of these waves satisfies

$v_{T_{\mathrm{i}}} \ll v_{\mathrm{p}} \ll v_{T_{\mathrm{e}}}$

where $v_{T \alpha}=\sqrt{k_{\mathrm{B}} T_{\alpha} / m_{\alpha}}$ is the thermal speed of either electron or ion species with $\alpha=\{e, i\}$, respectively, in which $k_{\mathrm{B}}$ is the Boltzmann constant, $T_{\alpha}$ and $m_{\alpha}$ are the temperature and mass, respectively. Thus, $\lambda_{\mathrm{FLR}} \ll 1$ (where $\lambda_{\mathrm{FLR}}$ is the finite
Larmor radius, FLR). However, because the condition $\lambda \gg \lambda_{D}$ (where $\lambda$ is the wave length, and $\lambda_{\mathrm{D}}$ is the Debye scale) is not always valid for the waves, charge separation effects may not be ignored, though the quasi-neutrality $\left(n_{\mathrm{e} 0} \approx n_{\mathrm{i} 0}\right.$ where $n_{\alpha 0}$ is the density at equilibrium) can still be applied because the space-charge density $n_{\mathrm{sc}}$, as a result of the charge separation, is still relatively small compared to $n_{\alpha 0}$. In a finite time, these space-charge density granulations retain their structural integrity and ballistically propagate along a specific direction to form "trains" of solitons propagating in space to form solitary waves (Pécseli, 1985; Chiueh and Diamond, 1986).

In order to provide the most basic picture for the emergence and propagation of nonlinear solitary waves, and thus to gain important insights into the effects of electron inertia on the features of solitary structures, while still being able to illustrate the process clearly, we focus on a system composed of two components: isothermal electrons and adiabatic ions. They are described by two-fluid equations under collision-free conditions in the Cartesian frame $(x, y, z)$, including conservation equations of mass, momentum, and energy, plus four Maxwell's equations. A generalized set of equations is as follows:

$$
\begin{aligned}
& \frac{\partial n_{\mathrm{e}}}{\partial t}+\nabla \cdot\left(n_{\mathrm{e}} \boldsymbol{u}_{\mathrm{e}}\right)=0 \\
& \frac{\partial n_{\mathrm{i}}}{\partial t}+\nabla \cdot\left(n_{\mathrm{i}} \boldsymbol{u}_{\mathrm{i}}\right)=0 \\
& \left(\frac{\partial}{\partial t}+\boldsymbol{u}_{\mathrm{e}} \cdot \nabla\right) \boldsymbol{u}_{\mathrm{e}}=-\frac{1}{m_{\mathrm{e}} n_{\mathrm{e}}} \nabla p_{\mathrm{e}}-\frac{e}{m_{\mathrm{e}}}\left(\boldsymbol{E}+\boldsymbol{u}_{\mathrm{e}} \times \boldsymbol{B}\right) \\
& \left(\frac{\partial}{\partial t}+\boldsymbol{u}_{\mathrm{i}} \cdot \nabla\right) \boldsymbol{u}_{\mathrm{i}}=-\frac{1}{m_{\mathrm{i}} n_{\mathrm{i}}} \nabla p_{\mathrm{i}}+\frac{e}{m_{\mathrm{i}}}\left(\boldsymbol{E}+\boldsymbol{u}_{\mathrm{i}} \times \boldsymbol{B}\right) \\
& p_{\mathrm{e}}=n_{\mathrm{e}} k_{\mathrm{B}} T_{\mathrm{e} 0} \\
& \frac{p_{\mathrm{i}}}{p_{\mathrm{i}} 0}=\left(\frac{n_{\mathrm{i}}}{n_{\mathrm{i} 0}}\right)^{\gamma} \\
& \nabla \times \boldsymbol{E}=-\frac{\partial \boldsymbol{B}}{\partial t} \\
& \nabla \times \boldsymbol{B}=\mu_{0} \boldsymbol{j}+\frac{1}{c^{2}} \frac{\partial \boldsymbol{E}}{\partial t} \\
& \nabla \cdot \boldsymbol{E}=\frac{\rho_{\mathrm{e}}}{\epsilon_{0}} \\
& \nabla \cdot \boldsymbol{B}=0
\end{aligned}
$$

where the coupling of the fluid variables to Maxwell's equataions occurs through the definitions that relate the particles' number densities $\left(n_{\mathrm{e}}, n_{\mathrm{i}}\right)$ and flow velocities $\left(\boldsymbol{u}_{\mathrm{e}}, \boldsymbol{u}_{\mathrm{i}}\right)$ to the current density $\left[\boldsymbol{j}=e\left(n_{\mathrm{i}} \boldsymbol{u}_{\mathrm{i}}-n_{\mathrm{e}} \boldsymbol{u}_{\mathrm{e}}\right)\right]$ and charge density $\left[\rho_{\mathrm{e}}=e\left(n_{\mathrm{i}}-n_{\mathrm{e}}\right)\right]$. Notice that the conservation equations of energy are reduced to an equation of state for electrons and an adiabatic equation for ions, respectively. The basic unknowns in the model are $n_{\mathrm{e}}, n_{\mathrm{i}}, \boldsymbol{u}_{\mathrm{e}}, \boldsymbol{u}_{\mathrm{i}}, \boldsymbol{E}$, and $\boldsymbol{B}$, while $T_{\mathrm{e} 0}$ is the equilibrium temperature of electrons, and $\gamma$ is the adiabatic index. Parameters $\epsilon_{0}$ and $\mu_{0}$ are the absolute permittivity and permeability in free space, respectively, and $c=1 / \sqrt{\epsilon_{0} \mu_{0}}$ is the vacuum speed of light. Hereafter, subscript "0" attached to parameters in English indicates "equilibrium". Note that $\boldsymbol{B}$ is different from $\boldsymbol{B}_{\text {ext }}$.

For convenience, we use dimensionless parameters: the electron and ion densities $n_{\mathrm{e}}$ and $n_{\mathrm{i}}$ are normalized by $n_{0}$; coordinates $\boldsymbol{r}=\{\mathrm{x}, \mathrm{y}, \mathrm{z}\}$ by electron Debye length $\lambda_{\mathrm{De}}=v_{\mathrm{T}_{\mathrm{e}}} / \omega_{\mathrm{pe}}$; velocity $\boldsymbol{u}_{\alpha}=\left\{u_{\alpha \mathrm{x}}, u_{\alpha \mathrm{y}}, u_{\alpha \mathrm{z}}\right\}$ by the acoustic 
speed $c_{\mathrm{S}}=\sqrt{k_{\mathrm{B}} T_{\mathrm{e} 0} / m_{\mathrm{i}}}$; pressure $p_{\alpha}$ by $p_{\alpha 0}=n_{0} k_{\mathrm{B}} T_{\alpha 0}$; time $t$ by ion plasma period $\tau_{\mathrm{pi}}=\omega_{\mathrm{pi}}^{-1}$ (notice that $\omega_{\mathrm{pi}} \lambda_{\mathrm{De}}=c_{\mathrm{s}}$ ); magnetic field $\boldsymbol{B}$ by a pseudo-magnetic field $B_{0}=m_{\mathrm{e}} \omega_{\mathrm{pi}} / e$; electric field $\boldsymbol{E}$ by a pseudo-electric field $E_{0}=c_{\mathrm{s}} B_{0}$; and, three coefficients are introduced: $\xi_{\mathrm{m}}=m_{\mathrm{i}} / m_{\mathrm{e}}, \xi_{\mathrm{T}}=T_{\mathrm{e} 0} / T_{\mathrm{i} 0}$, and $\xi_{\mathrm{v}}=v_{\mathrm{T}_{\mathrm{e}}} / c$. A dimensionless expression of Eq. (3) is

$$
\begin{aligned}
& \frac{\partial n_{\mathrm{e}}}{\partial t}+\nabla \cdot\left(n_{\mathrm{e}} \boldsymbol{u}_{\mathrm{e}}\right)=0 \\
& \frac{\partial n_{\mathrm{i}}}{\partial t}+\nabla \cdot\left(n_{\mathrm{i}} \boldsymbol{u}_{\mathrm{i}}\right)=0 \\
& \left(\frac{\partial}{\partial t}+\boldsymbol{u}_{\mathrm{e}} \cdot \nabla\right) \boldsymbol{u}_{\mathrm{e}}=-\xi_{\mathrm{m}} \nabla \ln n_{\mathrm{e}}-\left(\boldsymbol{E}+\boldsymbol{u}_{\mathrm{e}} \times \boldsymbol{B}\right) \\
& \left(\frac{\partial}{\partial t}+\boldsymbol{u}_{\mathrm{i}} \cdot \nabla\right) \boldsymbol{u}_{\mathrm{i}}=-\frac{1}{\xi_{\mathrm{T}}} \frac{\gamma}{\gamma-1} \nabla n_{\mathrm{i}}^{\gamma-1}+\frac{1}{\xi_{\mathrm{m}}}\left(\boldsymbol{E}+\boldsymbol{u}_{\mathrm{i}} \times \boldsymbol{B}\right) \\
& \nabla \times \boldsymbol{E}=-\frac{\partial \boldsymbol{B}}{\partial t} \\
& \nabla \times \boldsymbol{B}=\xi_{\mathrm{v}}^{2}\left(n_{\mathrm{i}} \boldsymbol{u}_{\mathrm{i}}-n_{\mathrm{e}} \boldsymbol{u}_{\mathrm{e}}\right)+\frac{\xi_{\mathrm{v}}}{\xi_{\mathrm{m}}} \frac{\partial \boldsymbol{E}}{\partial t} \\
& \nabla \cdot \boldsymbol{E}=\xi_{\mathrm{m}}\left(n_{\mathrm{i}}-n_{\mathrm{e}}\right) \\
& \nabla \cdot \boldsymbol{B}=0
\end{aligned}
$$

To reduce the complexity of math in solving the problem and pay close attention to the formation of solitary structures, we employ a slab model where all parameters depend only on the $\mathrm{x}$-coordinate. In this case, $\gamma=3$. By using a single independent variable $X$ for a self-similar transformation $X=x-M t$ (Lee and Kan, 1981), where $M$ is the Mach number independent of $X$, along with

$$
\frac{\partial}{\partial t}=-M \frac{\mathrm{d}}{\mathrm{d} X}, \quad \frac{\partial}{\partial x}=\frac{\mathrm{d}}{\mathrm{d} X}
$$

we obtain a set of equations as follows:

$$
\begin{aligned}
& \frac{\mathrm{d} u_{\mathrm{ex}}}{\mathrm{d} X}=-\left(E_{\mathrm{x}}+u_{\mathrm{ey}} B_{\mathrm{z}}-u_{\mathrm{ez}} B_{\mathrm{y}}\right) /\left(u_{\mathrm{ex}}-\frac{\xi_{\mathrm{m}}}{u_{\mathrm{ex}}}\right) \\
& \frac{\mathrm{d} u_{\mathrm{ey}}}{\mathrm{d} X}=-\left(S_{\mathrm{y}}-u_{\mathrm{ex}} B_{\mathrm{z}}+u_{\mathrm{ez}} B_{\mathrm{x} 0}\right) / u_{\mathrm{ex}} \\
& \frac{\mathrm{d} u_{\mathrm{ez}}}{\mathrm{d} X}=-\left(S_{\mathrm{z}}+u_{\mathrm{ex}} B_{\mathrm{y}}-u_{\mathrm{ey}} B_{\mathrm{x} 0}\right) / u_{\mathrm{ex}} \\
& \frac{\mathrm{d} u_{\mathrm{ix}}}{\mathrm{d} X}=\xi_{\mathrm{m}}^{-1}\left(E_{\mathrm{x}}+u_{\mathrm{iy}} B_{\mathrm{Z}}-u_{\mathrm{iz}} B_{\mathrm{y}}\right) /\left(u_{\mathrm{ix}}-\frac{3 S_{n}^{2}}{\xi_{\mathrm{T}} u_{\mathrm{ix}}^{3}}\right) \\
& \frac{\mathrm{d} u_{\mathrm{iy}}}{\mathrm{d} X}=\xi_{\mathrm{m}}^{-1}\left(S_{\mathrm{y}}-u_{\mathrm{ix}} B_{\mathrm{z}}+u_{\mathrm{iz}} B_{\mathrm{x} 0}\right) / u_{\mathrm{ix}} \\
& \frac{\mathrm{d} u_{\mathrm{iz}}}{\mathrm{d} X}=\xi_{\mathrm{m}}^{-1}\left(S_{\mathrm{z}}+u_{\mathrm{ix}} B_{\mathrm{y}}-u_{\mathrm{iy}} B_{\mathrm{x} 0}\right) / u_{\mathrm{ix}} \\
& \frac{\mathrm{d} E_{\mathrm{x}}}{\mathrm{d} X}=-\xi_{\mathrm{m}} n_{\mathrm{sc}} \\
& \frac{\mathrm{d} B_{\mathrm{y}}}{\mathrm{d} X}=\xi_{\mathrm{v}}^{2} S_{n}\left(\frac{u_{\mathrm{iz}}}{u_{\mathrm{ix}}}-\frac{u_{\mathrm{ez}}}{u_{\mathrm{ex}}}\right) /\left(1-\frac{\xi_{\mathrm{v}}^{2} M^{2}}{\xi_{\mathrm{m}}}\right) \\
& \frac{\mathrm{d} B_{\mathrm{z}}}{\mathrm{d} X}=-\xi_{\mathrm{v}}^{2} S_{n}\left(\frac{u_{\mathrm{iy}}}{u_{\mathrm{ix}}}-\frac{u_{\mathrm{ey}}}{u_{\mathrm{ex}}}\right) /\left(1-\frac{\xi_{\mathrm{v}}^{2} M^{2}}{\xi_{\mathrm{m}}}\right)
\end{aligned}
$$

in which

$$
\begin{aligned}
& S_{n}=u_{\mathrm{xx} 0}-M \\
& S_{\mathrm{y}}=E_{\mathrm{y} 0}-M B_{\mathrm{z} 0}, S_{\mathrm{z}}=E_{\mathrm{z} 0}+M B_{\mathrm{y} 0} \\
& n_{\mathrm{e}}=S_{n} / u_{\mathrm{ex}}, n_{\mathrm{i}}=S_{n} / u_{\mathrm{ix}} \\
& n_{\mathrm{sc}}=n_{\mathrm{e}}-n_{\mathrm{i}} \\
& E_{\mathrm{y}}=S_{\mathrm{y}}+M B_{\mathrm{z}}, \quad E_{\mathrm{z}}=S_{\mathrm{z}}-M B_{\mathrm{y}} \\
& B_{\mathrm{x}}=B_{\mathrm{x} 0}
\end{aligned}
$$

by using boundary conditions $\left.n_{\mathrm{e}}\right|_{X=0}=n_{0},\left.n_{\mathrm{i}}\right|_{X=0}=n_{0}$, $u_{\mathrm{xx} 0}=\left.u_{\mathrm{ex} 0}\right|_{X=0}=\left.u_{\mathrm{ix} 0}\right|_{X=0}=U_{0}-M,\left.\quad E_{\mathrm{y}}\right|_{X=0}=E_{\mathrm{y} 0}$, $\left.E_{\mathrm{z}}\right|_{X=0}=E_{\mathrm{z} 0},\left.B_{\mathrm{X}}\right|_{X=0}=B_{\mathrm{x} 0},\left.B_{\mathrm{y}}\right|_{X=0}=B_{\mathrm{y} 0}$, and $\left.B_{\mathrm{Z}}\right|_{X=0}=$ $B_{\mathrm{Z} 0}$. Note that the $u_{\alpha x}$-origin is shifted from " 0 " to " $M$ ", and the density equations and Gauss's law require $S_{\mathrm{ne}}=S_{\mathrm{ni}}$, written as $S_{n}$. Note that the charge density $\rho_{\mathrm{e}}$ in Eq. (3) is at present expressed by a space-charge density $n_{\mathrm{sc}}$ of solitary waves.

Equation (6) describes localized, coherent solitary waves which may be excited in the two-fluid system by the balance of nonlinearity and the dispersive effect (Davidson, 1972; Drazin, 1984, and references therein). They transport energy from nonlinear solitary waves to ambient plasma particles owing to their retained shape and speed during propagations (Davydov, 1985; Hasegawa and Kodama, 1995), superimposing upon background propagating or non-propagating plasma oscillations, such as IA and/or IC modes. The classical dispersion relations of these linear modes have widely been studied experimentally and theoretically since 1960s, e.g., Tanaca et al. (1966a, b, 1967; IA mode), Hirose et al. (1970a, b, c; IC mode). By solving the perturbed equations of Eq. (3), we present related dispersion relations in Appendices for use below.

We solve Eq. (6) in three cases by steps. We start from introducing a basic case of parallel-propagating, electrostatic IA solitary waves where all variables has only one component along $X$. Then, we expand the study to a more generalized case where they have normal three components. Based on these studies we focus on the evolving patterns of oscillitons to show the modulation of the electron inertia on lowfrequency solitary waves by introducing high-frequency oscillations into amplitudes.

\section{In the absence of electron inertia: parallel-propagating IA solitary waves}

When the electron inertia is neglected, the simplest nonlinear solitary wave is in the IA mode. This mode propagates in a direction parallel to local magnetic field lines, superimposing upon background linear electrostatic IA oscillations the dispersion relation of which is given in Appendix A.

The set of equations to describe nonlinear IA solitary waves propagating along $\boldsymbol{B}_{\mathrm{ext}}=B_{\mathrm{x} 0} \hat{\mathrm{e}}_{\mathrm{x}}$ can be derived from Eq. (6) as follows:

$$
\left.\begin{array}{l}
n_{\mathrm{e}}=e^{\Phi} \\
\frac{\mathrm{d} u_{\mathrm{ix}}}{\mathrm{d} X}=\frac{-\mathrm{d} \Phi / \mathrm{d} X}{u_{\mathrm{ix}}-\frac{3 S_{n}^{2}}{\xi_{T} u_{\mathrm{ix}}^{3}}} \\
\frac{\mathrm{d}^{2} \Phi}{\mathrm{d} X^{2}}=n_{\mathrm{Sc}}
\end{array}\right\}
$$

by using $n_{\mathrm{i}}=S_{n} / u_{\mathrm{ix}}, n_{\mathrm{e}}=S_{n} / u_{\mathrm{ex}}$, and $E_{\mathrm{x}} / \xi_{\mathrm{m}}=-\mathrm{d} \Phi / \mathrm{d} X$. Note that (1) all parameters are dimensionless, and $\Phi$ is the normalized electrostatic potential the unit of which is $k_{\mathrm{B}} T_{\mathrm{e} 0} / e$; (2) $S_{\mathrm{y}}=S_{\mathrm{z}}=0$ due to the fact only $E_{\mathrm{x}}$ component of $\boldsymbol{E}$ exists. 


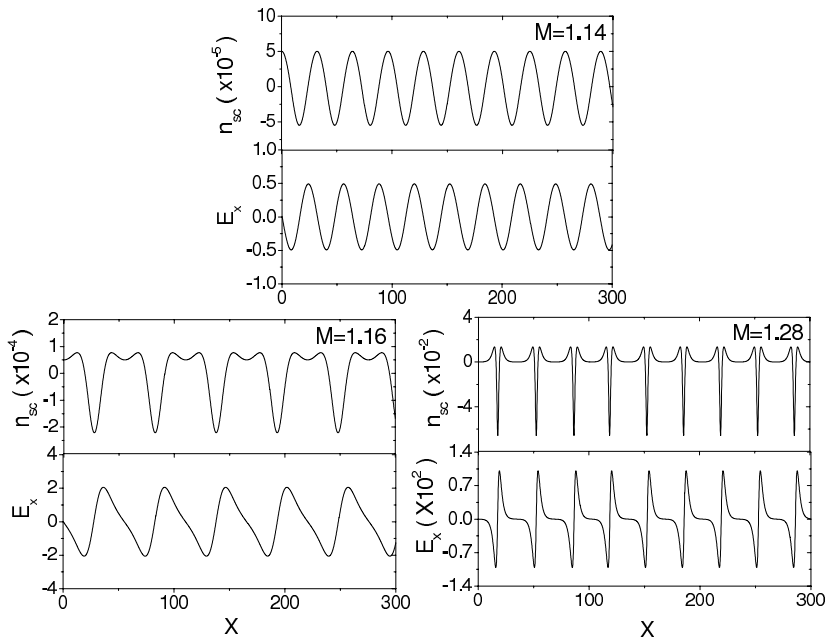

Fig. 2. Parallel-propagating IA soliton space-charge density $n_{\mathrm{sc}}$ and the wave-field $E_{\mathrm{X}}$ under $\xi_{\mathrm{T}}=10$, and $M=1.14$ (top panel), 1.16 (bottom left), 1.28 (bottom right), showing three types of shapes (sinusoidal, sawtooth, and spiky/bipolar), respectively.

Equation (8) produces a propagating IA mode satisfying (cf. Eq. 9 in Ma and Hirose, 2009a)

$$
\left.\begin{array}{l}
\frac{\mathrm{d}^{2} \Phi}{\mathrm{d} X^{2}}=e^{\Phi} \\
-\frac{\sqrt{2}}{\sqrt{\left(1+\frac{3}{\xi_{\mathrm{T}} M^{2}}-\frac{2 \Phi}{M^{2}}\right)+\sqrt{\left[\left(1+\frac{3}{\xi_{\mathrm{T}} M^{2}}-\frac{2 \Phi}{M^{2}}\right)\right]^{2}-\frac{12}{\xi_{\mathrm{T}} M^{2}}}}}
\end{array}\right\}
$$

from which, as well as Eq. (8), the space-charge density $n_{\mathrm{sc}}$ and the wave-field $E_{\mathrm{x}}$ of solitary waves can be solved numerically.

Take $\xi_{\mathrm{T}}=10$. The solitary structures are calculated under different Mach numbers. Their evolution is exhibited by $n_{\mathrm{sc}}$ and $E_{\mathrm{x}}$. Figure 2 illustrates an example of the features of the soliton trains under $M=1.14,1.16,1.28$, respectively.

The top two panels in the figure illustrate sinusoidal shapes of both $n_{\mathrm{sc}}$ and $E_{\mathrm{x}}$, respectively. They both have small amplitudes at $M=1.14: n_{\mathrm{sc}}$ is within $5 \times 10^{-5}$ and $E_{\mathrm{x}}$ is no larger than 0.5. By contrast, when $M$ increases to 1.16 , each packet of the former exposes a tooth shape, while that of the latter display a sawtooth one, visible in the two bottom left panels. When the Mach number continues to increase to 1.28, the two bottom right panels disclose spiky shapes, unipolar for $n_{\mathrm{sc}}$ and bipolar for $E_{\mathrm{x}}$, respectively. It deserves to mention again that these three shapes of IA $E_{\mathrm{x}}$ structures (i.e., sinusoidal, sawtooth, and spiky/bipolar) are well-known in satellite observations. They were first reported by S3-3 satellite in late 1970s (Temerin et al., 1979) and have thereafter been detected in various regions in geospace by numerous satellites (see a detailed review in Ma and Hirose, 2009a).

In order to know the features in the frequency regime, we make use of a fast Fourier transform (FFT) algorithm to give the power densities of the three $E_{\mathrm{x}}$ waveforms, as shown

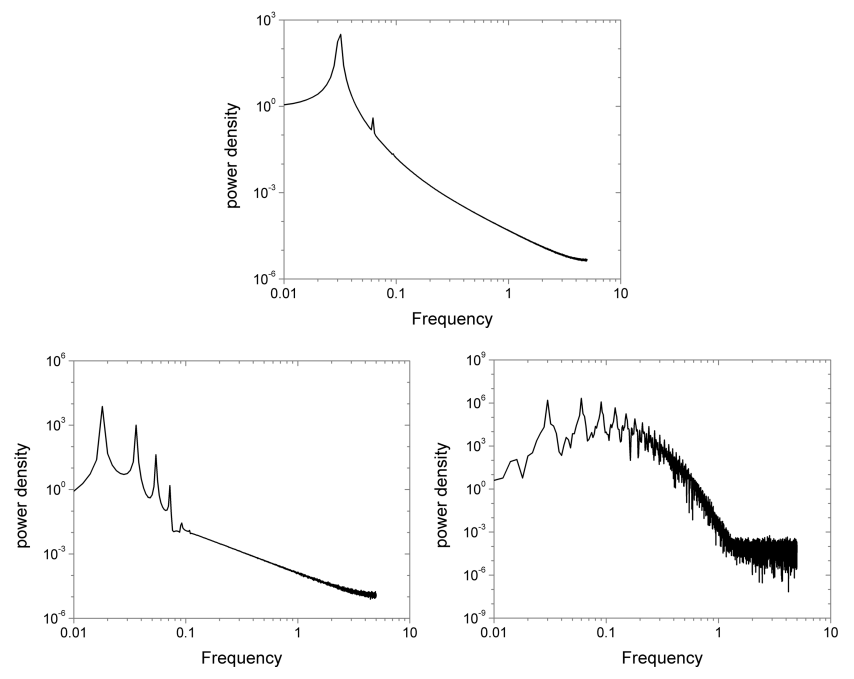

Fig. 3. FFT spectra of $E_{\mathrm{X}}$ in corresponding panels of Fig. 2.

in Fig. 3 where the vertical axis has an arbitrary unit. The top panel is the spectrum at $M=1.14$, exhibiting a couple of peaks: one is at $\omega_{1}=0.031 \omega_{\mathrm{pi}}$, the fundamental frequency of the imperfect sine wave; the other is at $\omega_{2}=0.062 \omega_{\mathrm{pi}}$, a harmonic of $\omega_{1}$. The former corresponds to the IA period $T_{X}=\omega^{-1}=32.3 \tau_{\mathrm{pi}}$ (note that $\omega_{\mathrm{pi}} \tau_{\mathrm{pi}}=1$ as assumed in normalizations) of the solitary structures along $X$, as provided in the top $E_{\mathrm{X}}$ panel of Fig. 2. As a double-check, we use Eq. (A4) to calculate the IA wavelength $k_{\mathrm{x}}$. In dimensionless units, the equation becomes

$\omega^{2}=\left(1+\frac{\gamma}{\xi_{\mathrm{T}}^{2}}\right) k_{\mathrm{x}}^{2}$

where the units of $\omega$ and $k_{\mathrm{x}}$ are $\omega_{\mathrm{pi}}$ and $\lambda_{\mathrm{De}}^{-1}$, respectively. In our calculations, we use $\xi_{\mathrm{T}}=10$. Thus, we have

$\omega=1.015 k_{\mathrm{x}}, \quad$ or, $\quad \lambda_{\mathrm{x}}=\frac{1}{k_{\mathrm{x}}}=\frac{1.015}{\omega}=32.7$

which is the exact IA wavelength shown in Fig. 2.

This first study tells us, though very basic, that in the nonlinear system, solitary waves will develop harmonics due to the fact that the structures do not have pure sine-waveforms. It is thus predictable that more harmonics will be developed if the shape of solitons goes farther away from a sine wave. This case is very similar to that of a piece of real musical instruments: in addition to the fundamental frequency $f$, a soliton train also has frequencies which are exact multiples of $f: f, 2 f, 3 f, \ldots$. This prediction is confirmed by other two panels of Fig. 3. At $M=1.16$, for example, the IA frequency spectrum of the sawtooth-like $E_{\mathrm{X}}$ structures have distinct four harmonics: $0.018,0.036,0.054$, and 0.072 , accompanied by a broadband of noises up to $5 \omega_{\mathrm{pi}}$. In the $M=1.28$ panel, bipolar solitons provide more harmonics, and these harmonics merge into the background noises eventually. Notice that in this case, it is not the fundamental frequency but 
the second harmonic that has the maximum power density. This warns us that, in data analysis to identify in-situ waves, a frequency with the strongest peak may not represent that the wave is excited at that frequency. This is important when determining signatures of, e.g., LH waves.

\section{In the absence of electron inertia: generalized IA/IC solitary waves}

In reality, variables have three components. We generalize the case discussed above to see the features of the nonlinear waves propagating in space. In this case, the background electrostatic waves contain propagating IA waves, and nonpropagating IC waves which oscillate locally. Appendix B describes the dispersion relation.

Neglect the electron inertia again. The second and third equations of Eq. (6) produce

$$
\left.\begin{array}{l}
u_{\mathrm{ex}} B_{\mathrm{z}}-S_{\mathrm{y}}=u_{\mathrm{ez}} B_{\mathrm{x} 0} \\
u_{\mathrm{ex}} B_{\mathrm{y}}+S_{\mathrm{z}}=u_{\mathrm{ey}} B_{\mathrm{x} 0}
\end{array}\right\}
$$

which gives

$$
u_{\mathrm{ey}} B_{\mathrm{z}}-u_{\mathrm{ez}} B_{\mathrm{y}}=\frac{S_{\mathrm{y}}}{B_{\mathrm{x} 0}} B_{\mathrm{y}}+\frac{S_{\mathrm{z}}}{B_{\mathrm{x} 0}} B_{\mathrm{z}}
$$

and

$$
\left.\begin{array}{l}
\frac{u_{\mathrm{ey}}}{u_{\mathrm{ex}}}=\frac{B_{\mathrm{y}}}{B_{\mathrm{x} 0}}+\frac{S_{\mathrm{z}} / u_{\mathrm{ex}}}{B_{\mathrm{x} 0}} \\
\frac{u_{\mathrm{ez}}}{u_{\mathrm{ex}}}=\frac{B_{\mathrm{z}}}{B_{\mathrm{x} 0}}-\frac{S_{\mathrm{y}} / u_{\mathrm{ex}}}{B_{\mathrm{x} 0}}
\end{array}\right\}
$$

Substituting above expressions for the terms in Eq. (6) leads to a new set of equations as follows:

$$
\left.\begin{array}{rl}
\frac{\mathrm{d} u_{\mathrm{ex}}}{\mathrm{d} X} & =\frac{E_{\mathrm{x}}+\frac{S_{\mathrm{y}}}{B_{\mathrm{x} 0} 0} B_{\mathrm{y}}+\frac{S_{\mathrm{Z}}}{B_{\mathrm{x} 0}} B_{\mathrm{z}}}{\frac{\xi_{\mathrm{m}}}{u_{\mathrm{ex}}}} \\
\frac{\mathrm{d} u_{\mathrm{ix}}}{\mathrm{d} X} & =\frac{1}{\xi_{\mathrm{m}}} \frac{E_{\mathrm{x}}+u_{\mathrm{iy}} B_{\mathrm{z}}-u_{\mathrm{iz}} B_{\mathrm{y}}}{u_{\mathrm{ix}}-\frac{3 S_{n}^{2}}{\xi_{\mathrm{T}} u_{\mathrm{ix}}^{3}}} \\
\frac{\mathrm{d} u_{\mathrm{iy}}}{\mathrm{d} X} & =\frac{1}{\xi_{\mathrm{m}}} \frac{S_{\mathrm{y}}-u_{\mathrm{ix}} B_{\mathrm{z}}+u_{\mathrm{iz}} B_{\mathrm{x} 0}}{u_{\mathrm{ix}}} \\
\frac{\mathrm{d} u_{\mathrm{iz}}}{\mathrm{d} X} & =\frac{1}{\xi_{\mathrm{m}}} \frac{S_{\mathrm{z}}+u_{\mathrm{ix}} B_{\mathrm{y}}-u_{\mathrm{iy}} B_{\mathrm{x} 0}}{u_{\mathrm{ix}}} \\
\frac{\mathrm{d} E_{\mathrm{x}}}{\mathrm{d} X} & =\xi_{\mathrm{m}} S_{n}\left(\frac{1}{u_{\mathrm{ix}}}-\frac{1}{u_{\mathrm{ex}}}\right) \\
\frac{\mathrm{d} B_{\mathrm{y}}}{\mathrm{d} X}=\xi_{\mathrm{v}}^{2} S_{n} \frac{\frac{u_{\mathrm{iz}}}{u_{\mathrm{ix}}}-\frac{B_{\mathrm{Z}}}{B_{\mathrm{x} 0}}+\frac{S_{\mathrm{y}} / u_{\mathrm{ex}}}{B_{\mathrm{x} 0}}}{1-\frac{\xi_{\mathrm{v}}^{2} M^{2}}{\xi_{\mathrm{m}}}} \\
\frac{\mathrm{d} B_{\mathrm{z}}}{\mathrm{d} X}=-\xi_{\mathrm{v}}^{2} S_{n} \frac{\frac{u_{\mathrm{i}}}{u_{\mathrm{ix}}}-\frac{B_{\mathrm{y}}}{B_{\mathrm{x} 0}}-\frac{S_{\mathrm{z}} / u_{\mathrm{ex}}}{B_{\mathrm{x} 0}}}{1-\frac{\xi_{\mathrm{v}}^{2} M^{2}}{\xi_{\mathrm{m}}}}
\end{array}\right\}
$$

Note that in the present case, the electron inertia is still not included, just as the previous case. Electrons thus continue to provide background conditions for the modulation of ions driven by space-charge electric fields to excite linear and nonlinear IA/IC waves. In simulations, five input parameters are chosen as follows: $M=1, m_{\mathrm{i}} / m_{\mathrm{p}}=16, \xi_{\mathrm{m}}=1836.2, \xi_{\mathrm{T}}=10$, $\xi_{\mathrm{v}}=0.1$. Various initial conditions are given to produce corresponding solitary structures. Deformed IA/IC solitary structures (sinusoidal, sawtooth, and spiky/bipolar) are obtained. An example is exposed in Fig. 4.

Figure 4 shows a sinusoidal IA/IC soliton train driven under boundary conditions: $U_{0}=\{0.3,0,0\}, E_{0}=\{0,0,1\}$, and $B_{0}=\{0.2,0,1\}$. Note that all the variables are restricted to the nonlinear evolutions along one self-similar X-coordinate in the slab model. In the figure, top left panel gives solitons' electron or ion density $\left(n_{\mathrm{e}}\right.$ or $\left.n_{\mathrm{i}}\right)$, and top right panel reveals the space-charge density $\left(n_{\mathrm{sc}}\right)$, inserted by an enlarged curve. Notice that the difference between the electron and ion densities is so small $\left(n_{\mathrm{sc}} \sim 10^{-5}\right)$, five orders smaller than either $n_{\mathrm{e}}$ or $n_{\mathrm{i}}$, that $n_{\mathrm{e}}$ and $n_{\mathrm{i}}$ curves are superimposed upon each other on the top left panel.

The middle three panels show three electric wave-field components $\left(E_{\mathrm{x}}, E_{\mathrm{y}}, E_{\mathrm{z}}\right)$. The $\mathrm{E}_{\mathrm{x}}$-panel demonstrates two types of oscillations: one is the IC mode in a larger period of $X \sim 170$ with a cyclotron frequency about $0.005-0.01 \omega_{\mathrm{pi}}$, and the other is the IA mode in a much smaller period of $X \sim 3$ with frequencies around $0.3 \omega_{\mathrm{pi}}$. Figure 5 gives a FFT spectrum of the $E_{\mathrm{X}}$-structures. Obviously, the IC mode has three harmonics at $f=0.0055 \omega_{\mathrm{pi}}, 0.011 \omega_{\mathrm{pi}}$, and $0.017 \omega_{\mathrm{pi}}$, respectively, while the IA mode shows a narrow-band spectrum within $0.3 \pm 0.05 \omega_{\mathrm{pi}}$. Above $0.6 \omega_{\mathrm{pi}}$ there occurs a wide band of noises.

Interestingly enough, the IA mode exist in neither $E_{\mathrm{y}}$ nor $E_{\mathrm{z}}$. Both of the components constitute a right (rather than left) circular polarization wave propagating along with the $E_{\mathrm{X}}$ wave, as shown in Fig. 6. The tip of the electric field vector perpendicular to $\hat{\boldsymbol{e}}_{\mathrm{X}}$ depicts a circle in the perpendicular plane, and describes a helix along the direction of wave propagation $X$. The magnitude of the electric field vector is constant as it rotates. This circular polarization is possible due to the fact that $E_{\mathrm{y}}$ and $E_{\mathrm{z}}$ are orthogonal with each other but have a same IC frequency.

Similar to $E_{\mathrm{y}}$ and $E_{\mathrm{z}}, B_{\mathrm{y}}$ and $B_{\mathrm{z}}$ give a pure IC mode, as depicted in the two bottom left panels of Fig. 4. They produce a propagating right circular polarization wave, while $B_{\mathrm{X}}$ keeps constant. The bottom right panel of the figure gives two total magnetic field strengths, the initial field $\left|B_{0}\right|$, and the soliton field $|B(X)|$. No doubt that the magnetic field strength is enhanced. This tells us that solitons carry a stronger magnetic field on average and thus store more magnetic energy. A direct consequence may attribute to the magnetic holes (or decreases, bubbles) in surrounding regions as observed by numerous satellites/spacecrafts (see, e.g., Tsurutani et al., 2005)

The other two distorted sawtooth and bipolar structures are exhibited in Figs. 7 and 8, respectively. The former is under boundary conditions: $U_{0}=\{0.2,0,0\}, E_{0}=\{0.5,0.5,1\}$, and $B_{0}=\{0.2,0.5,3\}$, while the latter is under boundary conditions: $U_{0}=\{0.3,0,0\}, E_{0}=\{0,0.4,1\}$, and $B_{0}=\{0.2,0,1\}$, however $M$ is reduced from 1 to 0.85 . There are a few similarities compared to the sinusoidal case. Firstly, $E_{\mathrm{y}}$ and $E_{\mathrm{z}}$ 


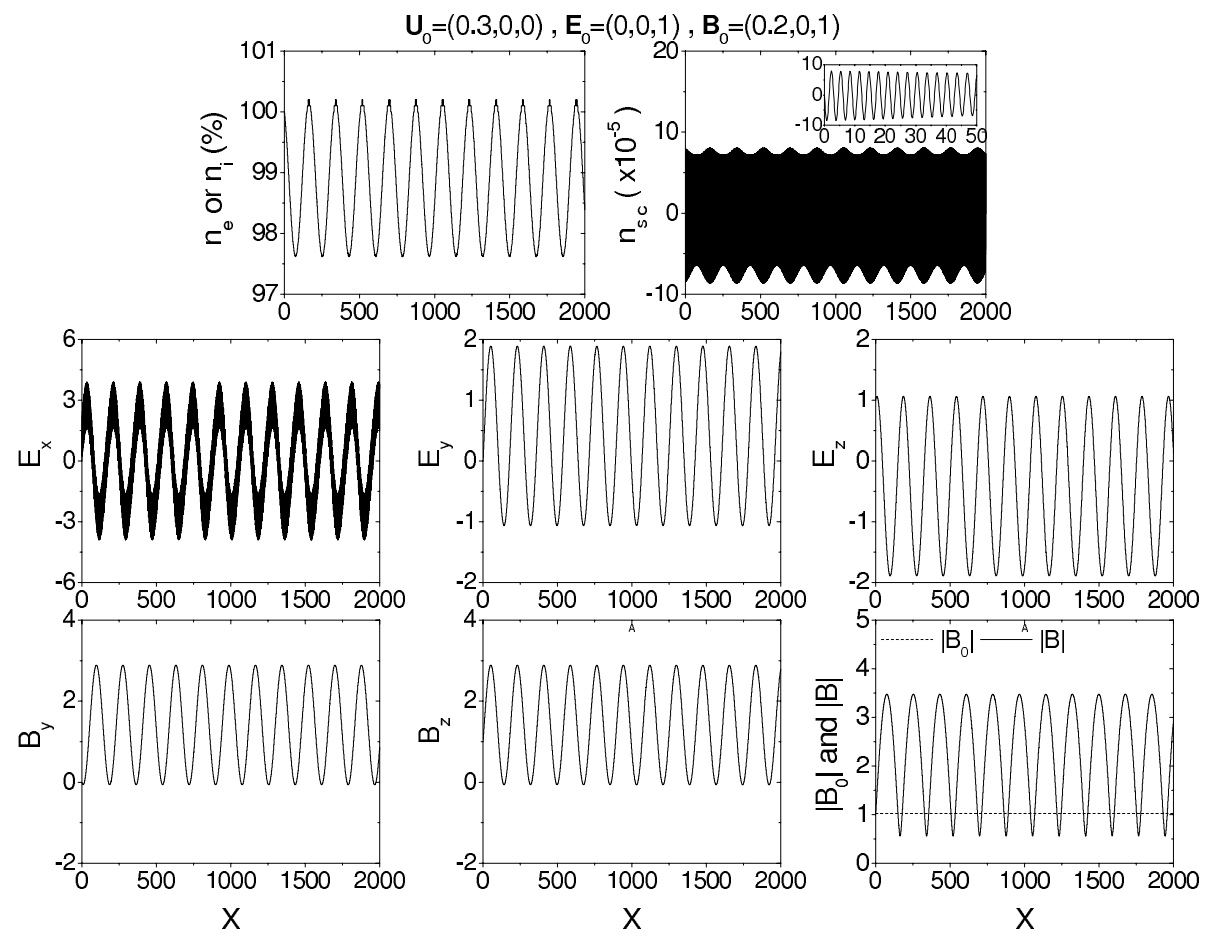

Fig. 4. Sinusoidal IA/IC solitary structures driven in a nonlinear system where all variables depend only on the $x$-coordinate (a slab model). Shown in panels of the figure are solitons' density ( $n_{\mathrm{e}}$ or $n_{\mathrm{i}}$; top left), space-charge density inserted by an enlarged curve ( $n_{\mathrm{sc}}$; top right), electric wave-field components $\left(E_{\mathrm{x}}, E_{\mathrm{y}}, E_{\mathrm{Z}}\right.$; middle row), magnetic wave-field components $B_{\mathrm{y}}$ (lower left) and $B_{\mathrm{Z}}$ (lower middle), and, total magnetic field strengths [initial $\left|B_{0}\right|$ and soliton $|B(X)|$; lower right]. Input parameters are as follows: $M=1, m_{\mathrm{i}} / m_{\mathrm{p}}=16, \xi_{\mathrm{m}}=1836.2, \xi_{\mathrm{T}}=10$, $\xi_{\mathrm{v}}=0.1$.

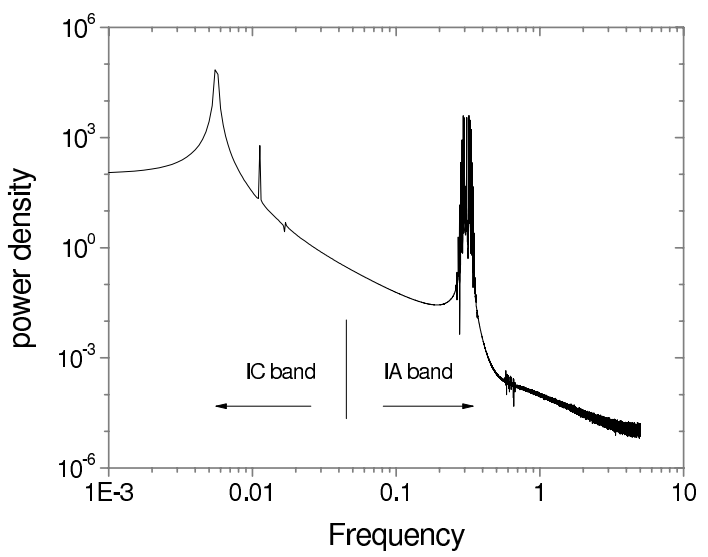

Fig. 5. FFT spectrum of the deformed sinusoidal $E_{\mathrm{X}}$ solitary structures.

form a right circular polarization wave; secondly, $B_{\mathrm{y}}$ and $B_{\mathrm{Z}}$ still hold a right polarization wave, however the sawtooth case offers an elliptical one, while the bipolar case is a circular one. Lastly, the total magnetic fields on average are all increased from their respective initial ones.

Nevertheless, the space-charge densities evolve very differently. Their amplitudes change much more abruptly but periodically with $X$. The period is about 500 in the sawtooth

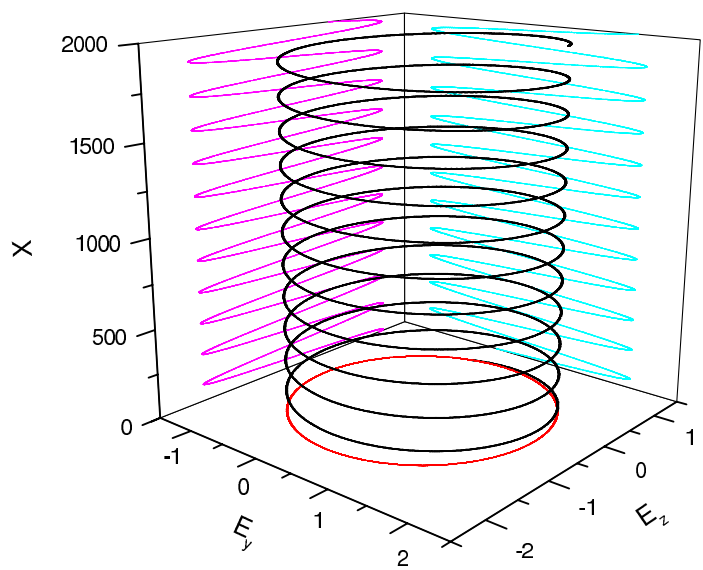

Fig. 6. Non-propagating circular polarization IC wave constituted by $E_{\mathrm{y}}$ and $E_{\mathrm{Z}}$ oscillations accompanying the sinusoidal $E_{\mathrm{X}}$ solitary structures.

case, while it is about 250 in the bipolar case. What is more, the maximum amplitude of the space-charge density does not retain constant in the latter case. There is a slight increase in $X$, while the pulsations have a higher-frequency ingredient: in the sawtooth case, the IA period in $X$ is several Debye lengths; in the bipolar case, the period in $X$ is only a few tenths of a Debye length. 


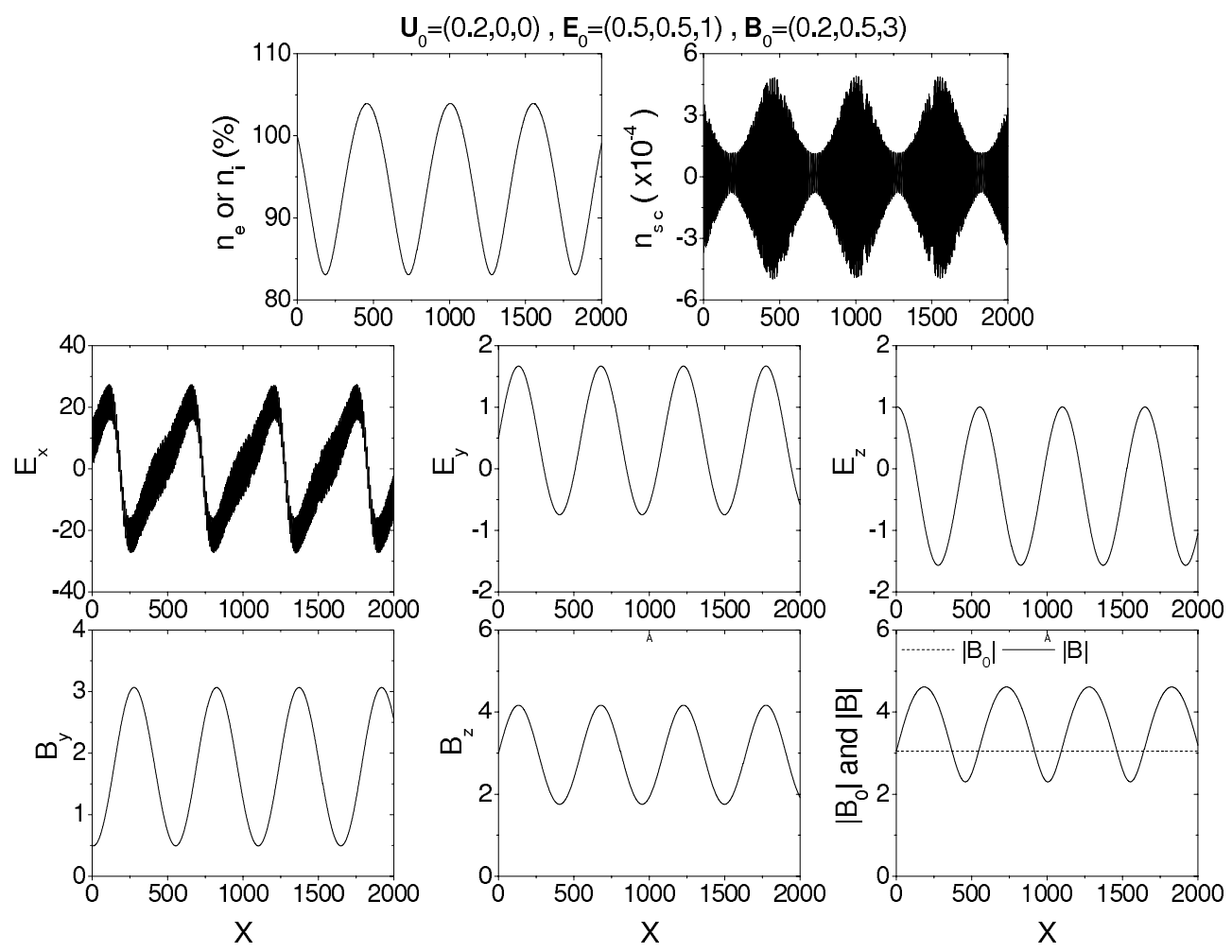

Fig. 7. Same as Fig. 4 but deformed sawtooth IA/IC solitary structures.

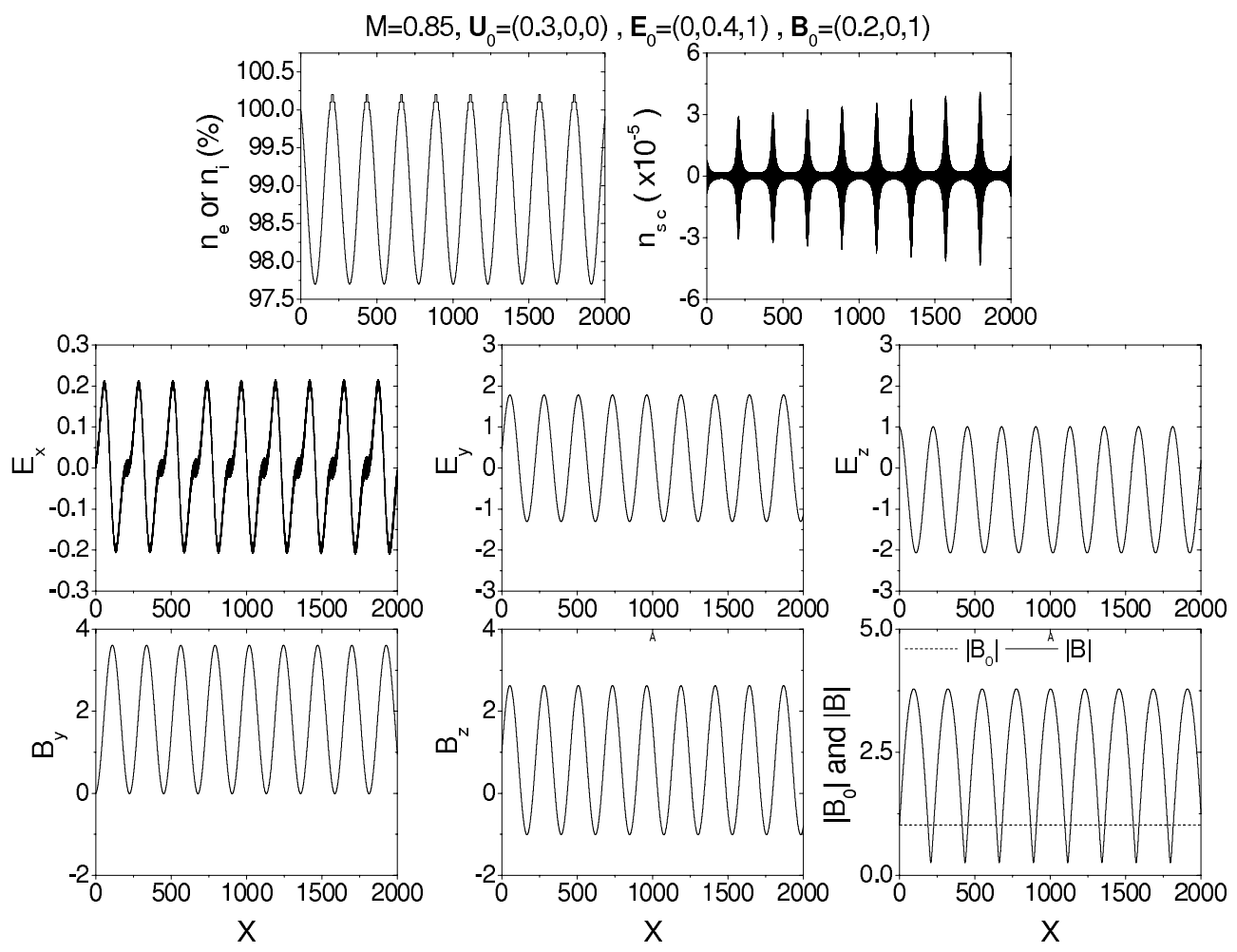

Fig. 8. Same as Fig. 4 but deformed bipolar IA/IC solitary structures. 

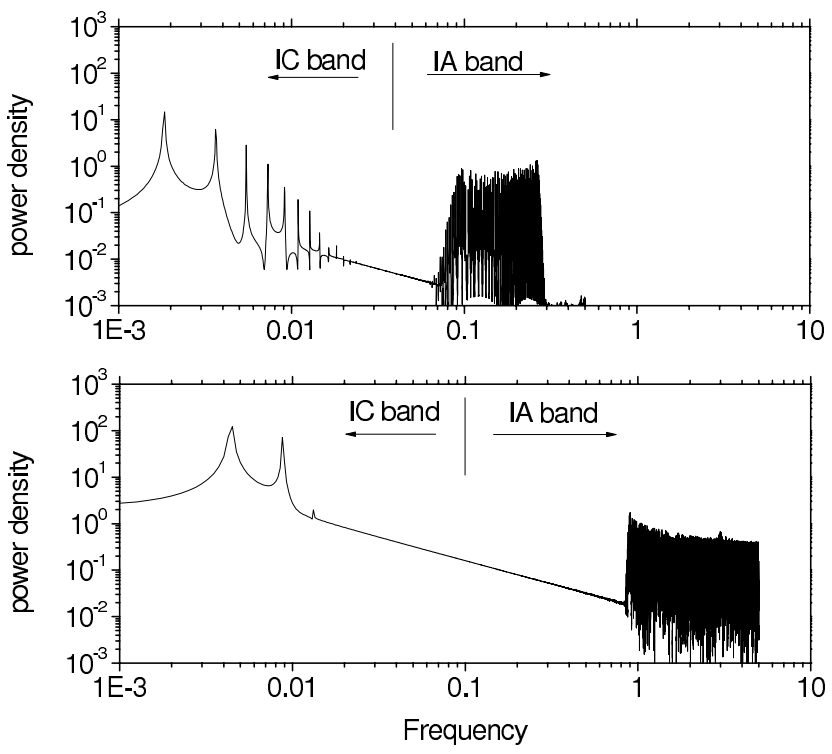

Fig. 9. FFT spectra of the sawtooth (upper panel) and bipolar (lower panel) $E_{\mathrm{X}}$ solitary structures.

Accordingly, the FFT spectra of the both cases expose different signatures, as shown in Fig. 9. In the sawtooth case (upper panel), the IC mode contains a series of harmonics but with a narrower IA band than the bipolar case (lower panel); by contrast, the bipolar case owns fewer IC harmonics, but with a broader IA band up to several $\omega_{\mathrm{pi}}$. This is understandable by considering the small wavelength of the IA mode: a few tenths of a Debye length in $X$ corresponds to several $\omega_{\mathrm{pi}}$ in frequency. As a double-check, let's turn to Eq. (B3). Its dimensionless expression is as follows:

$\omega^{2}=k^{2}\left(\frac{\gamma}{\xi_{\mathrm{T}}^{2}}+\frac{1}{1+k^{2}}\right)$

Obviously, when $k$ is large, the oscillation frequency cannot be smaller than the ion plasma frequency, i.e., $\omega \geq 1$. The critical wavenumber $k_{\mathrm{cr}}$ is

$k_{\mathrm{cr}}=\sqrt{\frac{1}{2}\left(\sqrt{1+\frac{4}{\gamma} \xi_{\mathrm{T}}^{2}}-1\right)}$

In our case, $\gamma=3$ and $\xi_{\mathrm{T}}=10$, leading to $k_{\mathrm{cr}}=2.3$ or $\lambda_{\mathrm{cr}}=k_{\mathrm{cr}}^{-1} \approx 0.4$. This means that if the wavelength is shorter than half of the electron Debye length, it is possible for IA oscillations to surpass the ion plasma frequency. This is interesting because it is well known that a LH frequency is always smaller than the ion plasma frequency. It is thus reasonable to deduce that IA peaks can exist in higher frequency band of a wave spectrum than LH ones, just as exhibited already by those observations in solar wind plasmas (see a comprehensive review by Briand, 2009).

\section{In the presence of electron inertia: LH "oscillitons"}

Above two sections discuss cases where the electron inertia is neglected. We have known that in those cases IA/IC solitary waves are able to be excited. In this section, we take into account the electron inertia, that is, electrons no longer response to the space-charge density instantly, but in a style constrained by both ion and electron kinetics. We must solve Eq. (6) directly, where both ion and electron masses play an equally important role. The shapes of solitary structures may be modulated to unexpected appearances which are different from the three conventional ones in either IA or IA/IC modes.

In the linear regime, a mega-amount of theoretical and experimental work were performed in 1960s and 1970s on plasma instabilities and excited waves in the presence of electron inertia (see, e.g., Artsimovich, 1964; Alexeff et al., 1970; Hirose and Alexeff, 1972; Mikhailovskii, 1974). The important outcome is, instead of linear IC and/or IC/IA modes as described in Appendices A and B, high-frequency $\left(\omega>\Omega_{\mathrm{i}}\right) \mathrm{LH}$ modes are triggered. In a two-fluid model in the presence of plasma nonuniformities, the LH dispersion relation was found to be heavily dependent of the gradients in, e.g., plasma density (Ma and Hirose, 2009b); if the plasma is uniform, as described by the generalized set of equations, Eq. (3), Appendix C provides a generalized dispersion, Eq. (C16), from which either electron and ion plasma oscillations, or IC, IA, ion and electron upper-hybrid, and lower-hybrid modes can be obtained under different directions relative to local magnetic field lines.

In the nonlinear regime, the structures of solitary waves can be simulated by employing directly the two-fluid set of equations, Eq. (6), as well as Eq. (7). We use a group of reference initial conditions and input parameters as follows: $\left\{u_{\mathrm{xx} 0}, u_{\mathrm{y} 0}, u_{\mathrm{z} 0}\right\}=\{0.3,0,0\},\left\{E_{\mathrm{x} 0}, E_{\mathrm{y} 0}, E_{\mathrm{z} 0}\right\}=$ $\{0,0,1\},\left\{B_{\mathrm{x} 0}, B_{\mathrm{y} 0}, B_{\mathrm{z} 0}\right\}=\{0.2,0,1\}$. We perform a parameterized study with changes in $M, m_{\mathrm{i}} / m_{\mathrm{p}}, \xi_{\mathrm{m}}, \xi_{\mathrm{T}}$, and $\xi_{\mathrm{v}}$, to see modulations of these input parameters on typical structures of soliton trains in the presence of the electron inertia.

\subsection{Solitary structure of reference}

The same as the IA/IC case, following five input parameters are used to perform a typical simulation: $M=1, m_{\mathrm{i}} / m_{\mathrm{p}}=16$, $\xi_{\mathrm{m}}=1836.2, \xi_{\mathrm{T}}=10, \xi_{\mathrm{v}}=0.1$. The features of the solitary structures are shown in Figs. 10 and 11.

In Fig. 10, the top left panel exposes density depletions in either $n_{\mathrm{e}}$ or $n_{\mathrm{i}}$ of the soliton train in propagation. The depth of the cavity structures is on average $1.33 \%$, with a minimum $3.39 \%$, of the background plasma density. There are also two shoulders for every density holes/dips, which is about $10 \%$ of the depth. The top right panel shows the space-charge density which is, on average, $-2.8 \times 10^{-8}$, with a maximum $7.1 \times 10^{-5}$ and a minimum $-5.3 \times 10^{-5}$. Thus, the density holes has a tiny excess of positive charges as an arithmetic mean. 


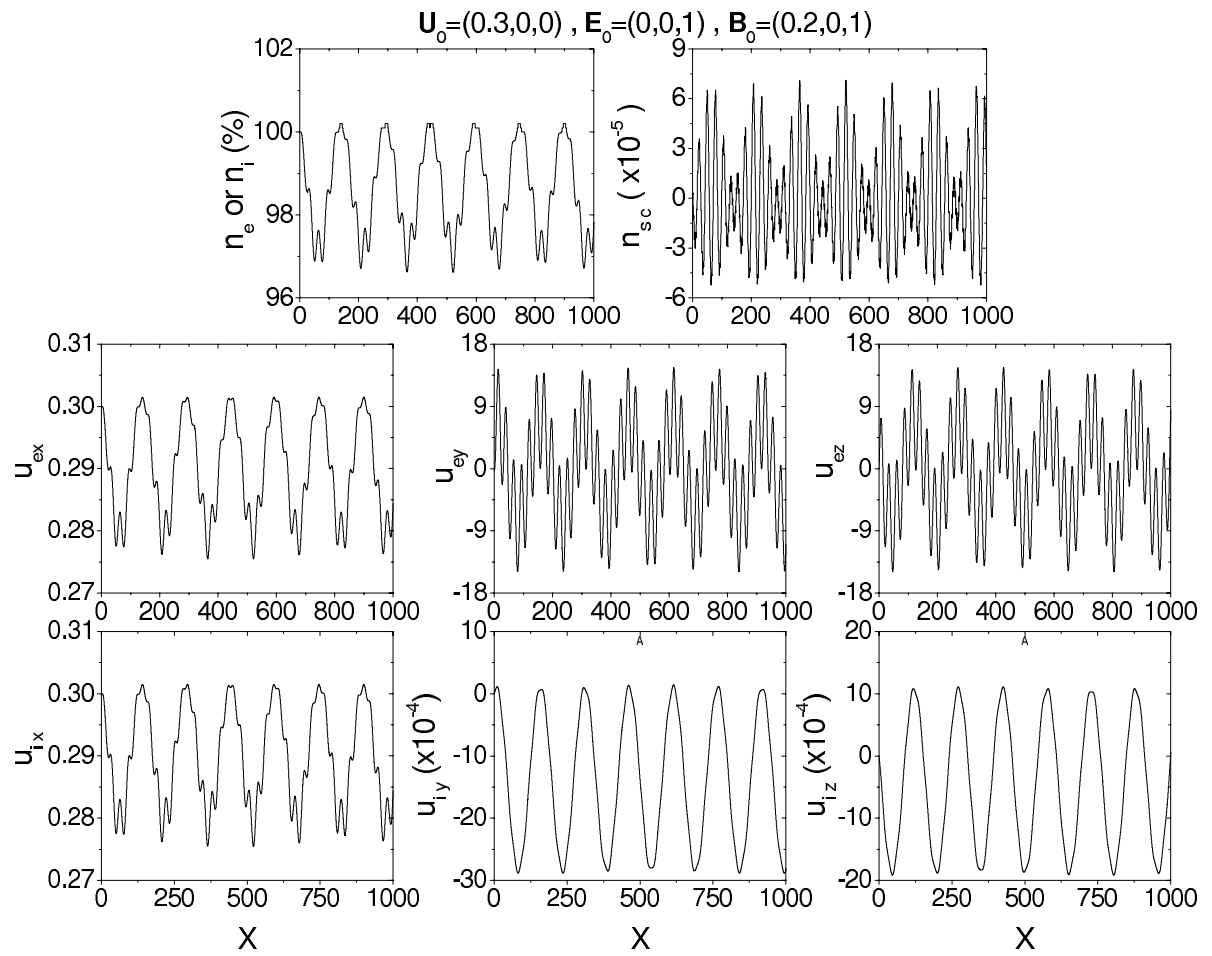

Fig. 10. Typical LH solitary structures. Shown in panels of the figure are solitons' density $\left(n_{\mathrm{e}}\right.$ or $n_{\mathrm{i}}$; top left), space-charge density $\left(n_{\mathrm{sc}}\right.$; top right), three components of electron velocity $\left(u_{\mathrm{ex}}, u_{\mathrm{ey}}, u_{\mathrm{ez}}\right.$; middle row), and that of ion velocity $\left(u_{\mathrm{ix}}, u_{\mathrm{iy}}, u_{\mathrm{iz}}\right.$; bottom row). Initial conditions and input parameters as follows: $\left\{u_{\mathrm{xx} 0}, u_{\mathrm{y} 0}, u_{\mathrm{z} 0}\right\}=\{0.3,0,0\},\left\{E_{\mathrm{x} 0}, E_{\mathrm{y} 0}, E_{\mathrm{z} 0}\right\}=\{0,0,1\},\left\{B_{\mathrm{x} 0}, B_{\mathrm{y} 0}, B_{\mathrm{z} 0}\right\}=\{0.2,0,1\} ; M=1$, $m_{\mathrm{i}} / m_{\mathrm{p}}=16, \xi_{\mathrm{m}}=1836.2, \xi_{\mathrm{T}}=10, \xi_{\mathrm{v}}=0.1$.

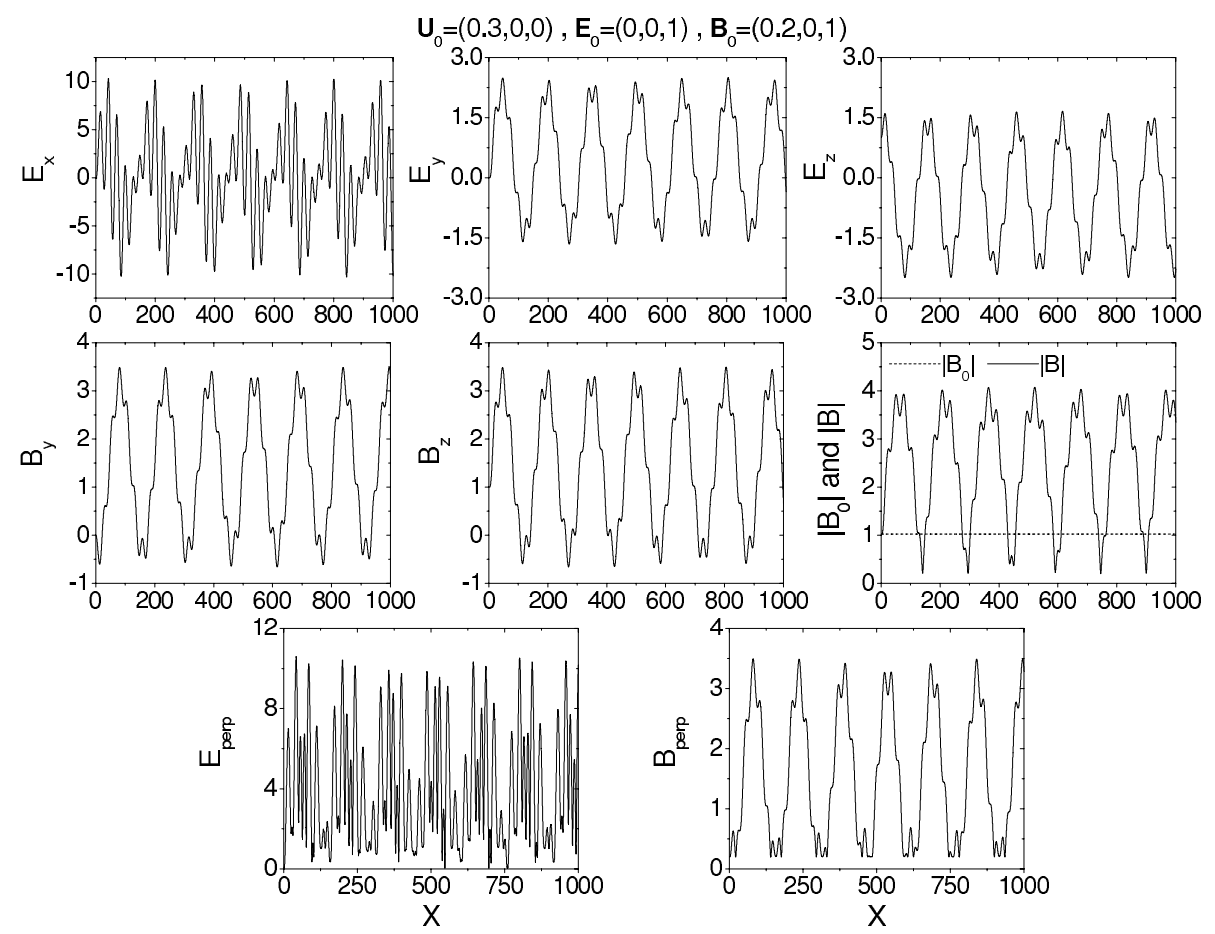

Fig. 11. Same as Fig. 10, however, shown in panels are the three components of electric wave-field ( $E_{\mathrm{X}}, E_{\mathrm{y}}, E_{\mathrm{Z}}$; top row); magnetic wavefield components $\left(B_{\mathrm{y}}\right.$ and $\left.B_{\mathrm{Z}}\right)$ and total strengthes $\left(\left|B_{0}\right|\right.$ and $\left.|B|\right)$ (middle row); and amplitudes of electric and magnetic fields in the plane perpendicular to the propagation direction $\left(E_{\perp}\right.$ and $B_{\perp}$, lower row), respectively. 
The X-dependent periodicity is also expressed by both electron and ion velocities. See the panels in the middle and lower rows. All the velocity components have a same period as that of the densities. Note that in the propagation direction, electrons and ions have a same speed $\left(u_{\mathrm{ex}}=u_{\mathrm{ix}}\right)$, while the perpendicular components of the ion velocity is much smaller than those of the electron velocity. This implies we can neglect the perpendicular motion of ions; equivalently, it is reasonable to assume that the velocity of ions have only one component which is in the propagation direction.

Figure 11 displays the three components of electric wavefield ( $E_{\mathrm{x}}, E_{\mathrm{y}}, E_{\mathrm{z}}$; top row); the magnetic wave-field components $\left(B_{\mathrm{y}}\right.$ and $\left.B_{\mathrm{z}}\right)$ and total strengthes $\left(\left|B_{0}\right|\right.$ and $\left.|B|\right)$ (middle row); and amplitudes of electric and magnetic fields in the plane perpendicular to the propagation direction $\left(E_{\perp}\right.$ and $B_{\perp}$, lower row), respectively. Similar to the IA/IC case, the two perpendicular components of both the electric and magnetic fields produce right circular polarization IC waves, and the total magnetic field strength of the solitary structures becomes stronger on average than the initial values. It deserves to mention that although every bipolar $E_{\mathrm{x}}$ structure contains a series of pulses, the averaged amplitude is zero. This means that along the propagation direction, there is no net potential drop carried by the soliton train. On the contrary, in the transverse plane, there always exists a pulsative electric field $E_{\perp}$ (lower left panel). Thus, the soliton train drives a $\boldsymbol{E}_{\perp} \times \boldsymbol{B}_{\mathrm{X}}$ drift which behaves as a prime mover of transverse ion heating in the presence of a crossed $B_{\mathrm{X}}$ (see details in Ma et al., 2009). Note that the soliton's magnetic field also has three components, and $B_{\perp} \neq 0$, as shown in the lower right panel of Fig. 11. Because $E_{\mathrm{x}}$ is normal to $B_{\perp}$, the $\boldsymbol{E}_{\mathrm{x}} \times \boldsymbol{B}_{\perp}$ drift provides an additional source for transverse ion heating. The study of this issue is beyond the scope of this article and will be introduced in another paper.

Figures 10 and 11 demonstrate two kinds of oscillations: IC and LH modes. From discussions presented in above sections in IA/IC cases, we know that the IC mode differentiates one soliton from the other in the train after a solitary wave is excited. The frequency can be easily estimated from the humps or dips of parameter envelops. In the present case, the period is $X=154$, corresponding to an frequency of $0.0065 \omega_{\mathrm{pi}}$. This frequency can be easily trace out from the perpendicular speeds $u_{\text {iy }}$ and $u_{\text {iz }}$ (relative to the propagation direction) of ions in Fig. 10, because, relative to electrons, their mass is so big that it is hard for them to respond immediately to the high-frequency drive. On the contrary, electrons are decoupled from ions in the perpendicular plane and their $u_{\text {ey }}$ and $u_{\text {ez }}$ carry IC envelops which are modulated by any possible faster oscillations. Look at the related panels in the figure: there are 6 peaks in every envelop, with a period of about $X=25$. This is contributed by the LH mode the frequency of which can be easily identified from $u_{\text {ex }}$ or $u_{\text {ix }}$ waveforms which reveal so strong a coupling in the propagation direction between electrons and ions that both of them contribute together to a LH mode while experiencing IC os- cillations. Such packets, manifesting a low-frequency (IC) solitary structure but with high-frequency (LH) modulation in amplitudes, are called "LH oscillitons".

Unexpectedly, by enlarging the coordinates (e.g., changing the scale of $X$ from a maximum value of 1000 to 100 ) we find two extra phenomena: (1) IA oscillations of much smaller amplitudes are superimposed upon the LH oscillitons, and they have a small period, about $X \sim 2-5$. (2) Both LH and IA waveforms are deformed sine-styles in both amplitudes and periods. These factors should contribute IA peaks and/or noises in the high-frequency side of power spectra. To verify this point, we plot Fig. 12 to give the FFT power spectrum (lower panel) of the $E_{\mathrm{X}}$ component in Fig. 11, along with those of the $E_{\mathrm{y}}$ structures in the IA/IC case (upper left) and the present case (upper right) to identify frequency signatures. From discussions in the last section, we know that in the IA/IC mode, ions have only IC oscillations in y. Thus, the spectrum of $E_{\mathrm{y}}$ displays only a IC peak as shown in the upper-left panel. The peak is at $0.0065 \omega_{\mathrm{pi}}$, corresponding to a period of $X=154$. In the LH-oscilliton case, we know that the LH mode is excited by taking into account the effect of electron inertia. This will surely leads to peaks or noises in the LH band. See the upper-right $E_{\mathrm{y}}$ panel of the figure. At $f=0.032,0.038,0.044$, there are three peaks, corresponding to periods of $X=23,26,31$. This indicates that there are three LH oscillations in $\hat{\boldsymbol{e}}_{\mathrm{y}}$-direction. Notice the dominant peak which represents a period of $X=26$. This is in agreement with the data given in the last paragraph, "a period of about $X=25$ ". However, due to the weak coupling between electrons and ions in the transverse plane, the LH signature is not very as strong as that in the parallel direction as revealed by the lower $\mathrm{E}_{\mathrm{x}}$-FFT panel.

In this panel, there are three bands. The IC band is on the low-frequency side of the spectrum with three harmonic peaks: $f=0.0065,0.01325,0.01975$. Note that the last peak is in the LH band which contains two groups: One owns higher magnitude but lower frequencies with peaks at $f=0.01825,0.025,0.03175,0.03825,0.04475,0.05625$, sharing a same interval between adjacent frequencies, and the other has lower magnitude but higher frequencies with peaks at $f=0.06325,0.06975,0.0765$ of the same interval. On the high-frequency side of the spectrum, there exists a narrow IA band of $\sim 0.25-0.4$. This corresponds to periods of $X$ from $\sim 2.5-4$, the same range as that checked by enlarging the coordinates. By reviewing the $\mathrm{E}_{\mathrm{x}}$-panel in Fig. 11, the $\mathrm{E}_{\mathrm{X}}$-FFT panel discloses an important message: an oscilliton may carry a hidden higher-frequency mode which is unable to be discerned from the obvious two features of its own: low-frequency solitary envelop and high-frequency oscillation. In the LH-oscilliton case, for example, without the FFT analysis, it were impossible to perceive the IA mode.

By reviewing the FFT spectra in last and present sections, it is worthwhile to stress that it is the electron inertia that modulates IA/IC solitary waves into LH oscillitons. They carry low-frequency IC envelops the amplitude of which 


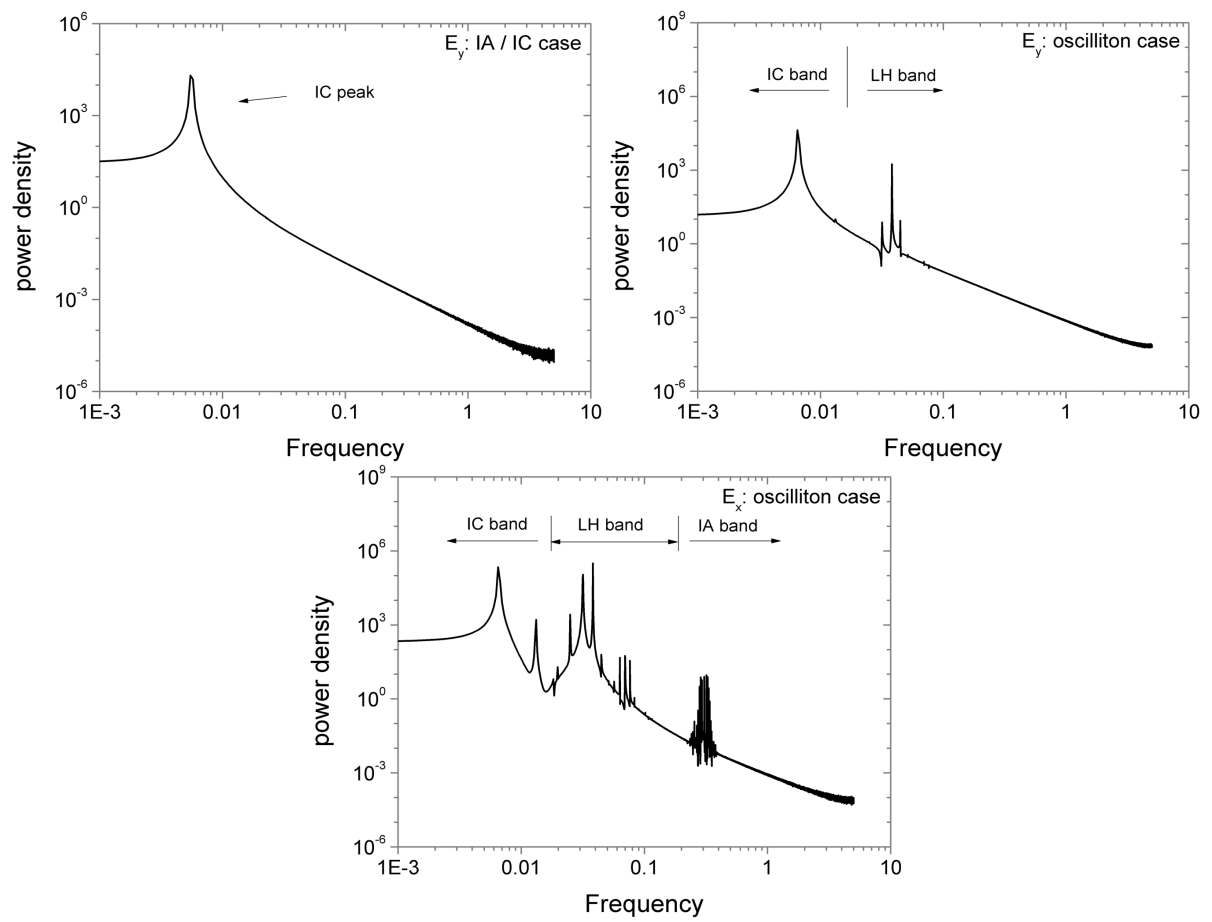

Fig. 12. FFT power spectra of socilliton structures. Upper left: $E_{\mathrm{y}}$ in the IA/IC case (last subsection); upper right: $E_{\mathrm{y}}$ in the oscilliton case (this subsection); lower panel: $E_{\mathrm{X}}$ in the ocilliton case (this subsection).

is embedded with high-frequency LH oscillations superimposed upon by a higher-frequency IA mode. Without the electron inertia, solitary structures do not have the modulated shapes (i.e., oscillitons) brought about by the interaction between LH and IA/IC ingredients, but exhibit only the traditional three (sinusoidal, sawtooth, and bipolar) IA/IC shapes.

\subsection{Parameterized simulation of oscilliton shapes}

There are several input parameters in Eqs. (6) and (7): Mach number $M$, mass ratio between ion and electron $\xi_{\mathrm{m}}$, temperature ratio $\xi_{\mathrm{T}}$, and speed ratio $\xi_{\mathrm{v}}$. Their magnitudes influence the appearance of oscilliton waves. Under the same initial conditions as the typical case discussed in the last section, we change the values of these parameters to expose their effect on the evolution of oscilliton trains.

Figure 13 exhibits the impact of $M$ on the LH oscillitons discussed in the last Section with $M=1$ (lower left 2 panels). We display four cases together for $M=0.85,0.9,1$, and 1.2 to see the evolution of space-charge density $n_{\mathrm{sc}}$ and electric wavefield $\mathrm{E}_{\mathrm{x}}$-component. With the increase of $M$, the amplitudes of both $n_{\mathrm{sc}}$ and $E_{\mathrm{X}}$ are enhanced. So does the number of solitons, indicating a reduced IC period. However, the frequency of the oscillations in every oscilliton is decreased. Above $M=1.2$, no oscilliton entities occur, meaning there is a limit for the Mach number only within which can oscillitons be driven.
Figure 14 exposes the impact of $\xi_{\mathrm{m}}=m_{\mathrm{i}} / m_{\mathrm{e}}$ on the LHoscilliton wave discussed in the last section with $m_{\mathrm{i}} / m_{\mathrm{p}}=16$ ( $m_{\mathrm{p}}$ is the proton mass; lower right 2 panels). We show four cases together for $m_{\mathrm{i}} / m_{\mathrm{p}}=3,4,8$, and 16 . When the ratio becomes larger, the amplitudes of both $n_{\mathrm{sc}}$ and $E_{\mathrm{x}}$ tend to smaller, while the solitary $E_{\mathrm{X}}$ structures change more from bipolar shapes to oscillitons, along with higher-frequency oscillations within every envelop. It is thus predictable that small mass ratios seem to restrain the electron inertia from exciting the LH mode in solitary waves. Different from the previous case, $\xi_{\mathrm{m}}$ does not affect the IC-period of the solitary waves.

Figure 15 demonstrates the influence of $\xi_{\mathrm{T}}=T_{\mathrm{e}} / T_{\mathrm{i}}$ on the LH-oscilliton wave discussed in the last section with $T_{\mathrm{e}} / T_{\mathrm{i}}=10$ (upper right 2 panels). We show four cases together for $T_{\mathrm{e}} / T_{\mathrm{i}}=6.5,10,15$, and 20 . The larger the ratio, the higher the amplitudes of the two parameters in roughly linear relations, respectively: $\left.n_{\mathrm{sc}}\right|_{\max }=3.6 \xi_{\mathrm{T}}-0.08 \xi_{\mathrm{T}}^{2}-18$, and $\left.E_{\mathrm{x}}\right|_{\max }=2.2 \xi_{\mathrm{T}}-0.04 \xi_{\mathrm{T}}^{2}-10$. We are concerned most about these relations because of the fact that the temperature ratio changes violently in space plasmas both spatially and temporally. Luckily, the plots simulated here expose that, except the amplitudes, the ratio changes neither the envelops nor the IC and LH periods.

Figure 16 illustrates the control of the electron thermal speed $V_{T_{e}}$ over oscillitons. When the speed is below $0.03 c$, no oscillitons are driven but only LH waves carrying IA fluctuations. With the increase of the speed, features of solitary 

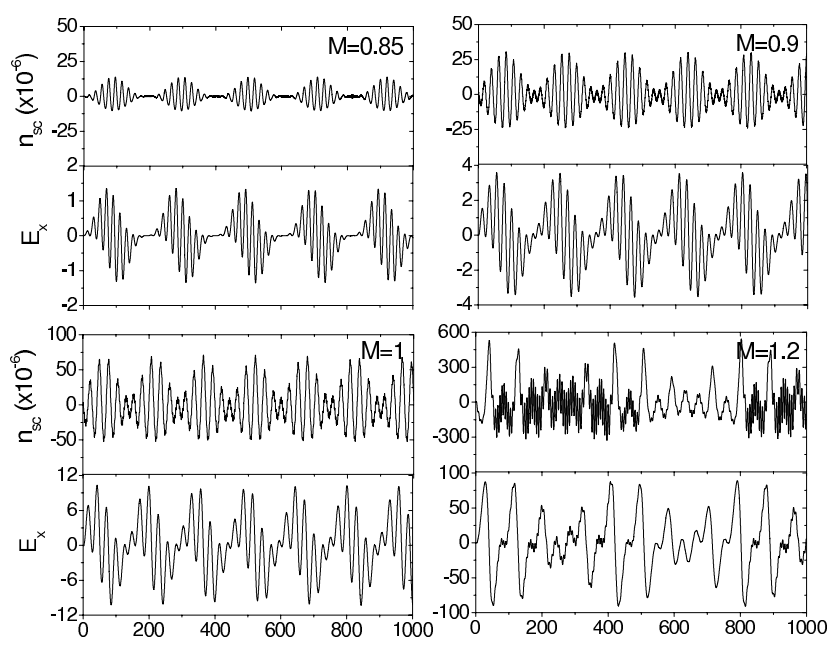

Fig. 13. Impact of the Mach number $M$ on LH-oscilliton waves. Only space-charge density $n_{\mathrm{c}}$ and electric wavefield $E_{\mathrm{X}}$-component are shown. Notice that the panels of $M=1$ is the typical case discussed in the last section.
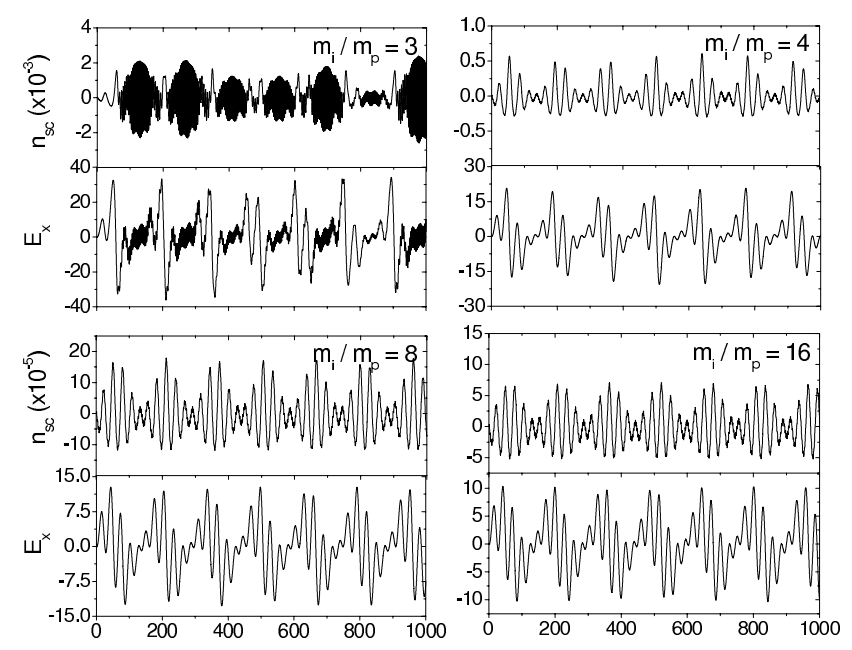

Fig. 14. Same as Fig. 13 but the impact of $\xi_{\mathrm{m}}=m_{\mathrm{i}} / m_{\mathrm{e}}$ on LHoscilliton waves. Notice that the panels of $m_{\mathrm{i}} / m_{\mathrm{e}}=16$ is the typical case discussed in the last section.

trains become evident gradually. However, after $V_{\mathrm{T}_{\mathrm{e}}}=0.13$, only bipolar waveforms manifest. During the development, the LH-IA signature is dominant at first, but suppressed increasingly with weaker oscillation and more obvious IC features, even out of sight at last.

Figures 13-16 exhibit the variation of the oscilliton structures with the four input parameters in the X-space. Accordingly, this change can also be expressed in the frequency regime. Figure 17 gives two such examples for Figs. 13-16 by illustrating the features of the FFT power spectra. The upper two panels is the spectra of $E_{\mathrm{x}}$ in Fig. 13 under $M=0.85$ and 1, respectively; the lower two ones is that in Fig. 16 under $V_{\mathrm{T}_{\mathrm{e}}} / c=0.04$ and 0.1 , respectively. The different IC/LH/IA
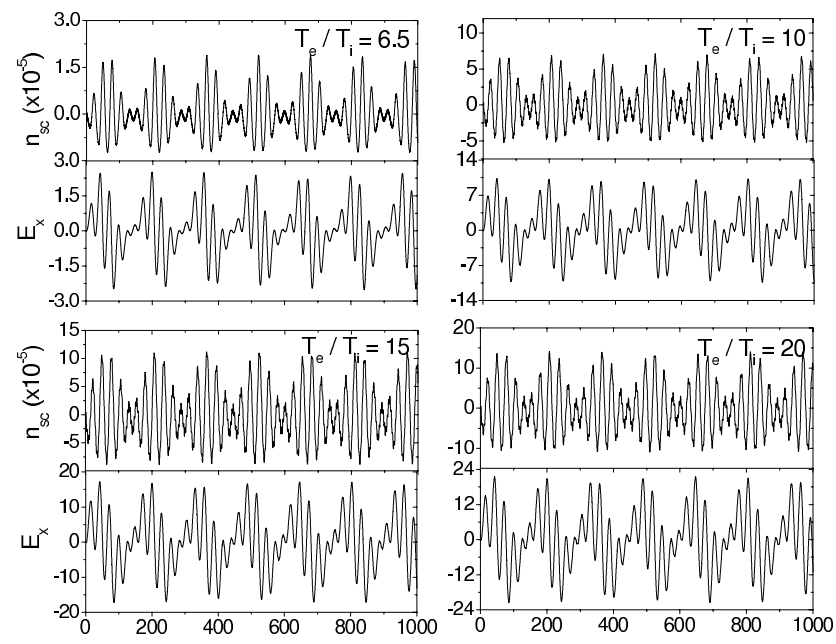

Fig. 15. Same as Fig. 13 but the impact of $\xi_{\mathrm{T}}=T_{\mathrm{e}} / T_{\mathrm{i}}$ on LHoscilliton waves. Notice that the panels of $T_{\mathrm{e}} / T_{\mathrm{i}}=10$ is the typical case discussed in the last section.
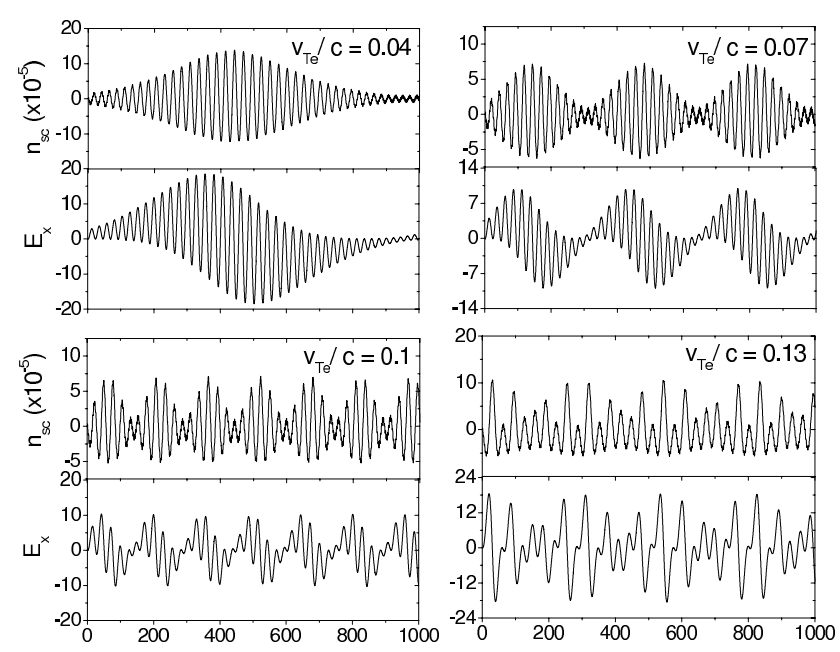

Fig. 16. Same as Fig. 13 but the impact of $\xi_{\mathrm{v}}=V_{\mathrm{T}_{\mathrm{e}}} / c$ on LHoscilliton waves. Notice that the panels of $V_{\mathrm{T}_{\mathrm{e}}} / c=0.1$ is the typical case discussed in the last section.

bands are identified by vertical dash lines in all panels. Evidently, on one hand, the two upper panels tell us that, at a larger Mach number, the IC band shifts to the right, indicating a decrease in frequency, while the LH band shifts to the left, meaning an increase in frequency. This is in agreement with what we have seen in Fig. 13. Unexpectedly, we can see a much weaker IA band ingredients appear at $M=1$, which is hard to be identified in Fig. 13. In the two lower panels, on the other hand, only the LH and IA bands exist at a smaller electron thermal speed; by contrast, if the speed becomes larger, the IC band is apparent, while the original LH/IA features are suppressed, with lower power densities and narrower bands. 

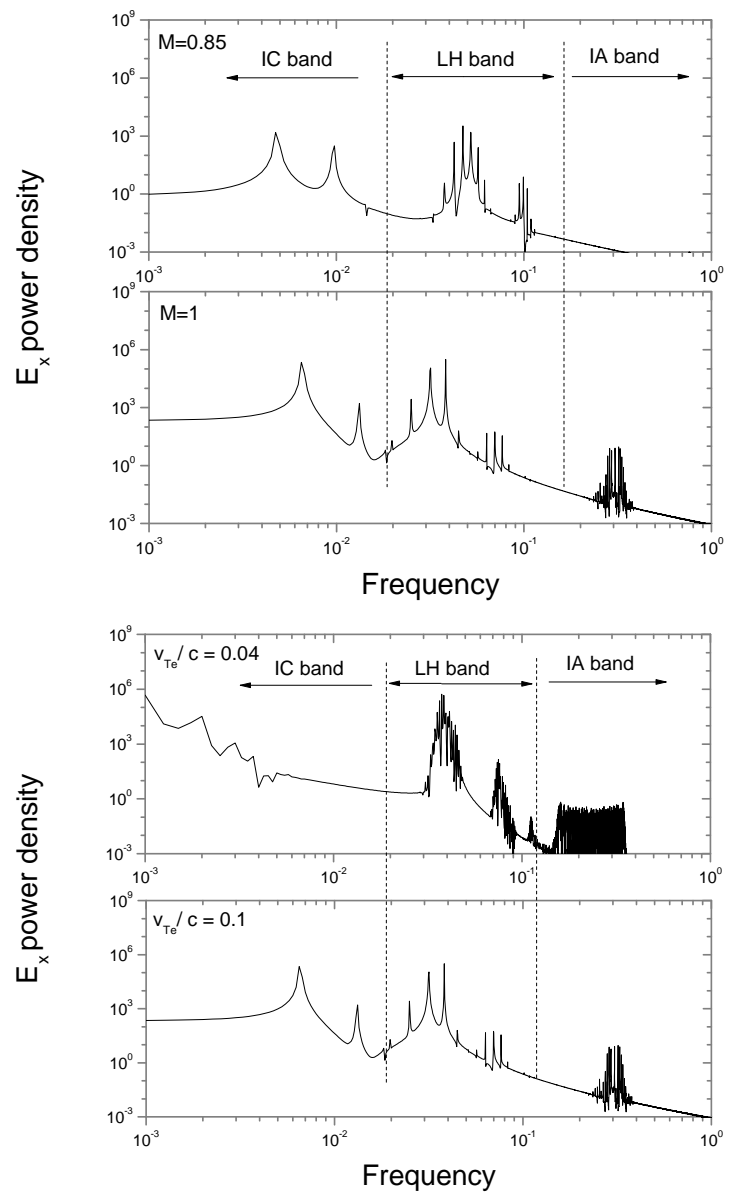

Fig. 17. FFT power spectra of $E_{\mathrm{X}}$ under two input parameters $M=0.85$ and 1 in Fig. 13 (left panels) and $V_{\mathrm{T}_{\mathrm{e}}} / c=0.04$ and 0.1 in Fig. 16, respectively, as two examples to illustrate the the variation of frequency with input parameters as exposed from Figs. 13 to 16. The different bands (IC/LH/IA) are labeled in all panels.

\subsection{A preliminary simulation to observations}

Though the excitation of oscillitons is known to be contributed by the electron inertia, and the modulation of the solitary structures by several input parameters is discussed, observations recorded much more complicated waveforms of oscilliton data. This attracts us to perform a preliminary datafit simulation.

Let's see the typical modulated waves measured by the Polar satellite (Cattell et al., 1998), as shown in Fig. 1. After 02:05:25.7, the slowly-oscillating envelop has a frequency $\omega_{\mathrm{s}} \sim 1 / 3$ ion gyrofrequency, while its amplitude was modulated violently by a quickly-oscillating ingredient with a frequency $\omega_{\mathrm{q}} \sim 8 \omega_{\mathrm{s}}$. The authors showed that the envelop of the modulated amplitude in each packet increases, reaching a maximum, and finally decreases with the period $1 / \omega_{\mathrm{s}}$, and the carried wave frequency $\omega_{\mathrm{q}}$ is close to the LH frequency.
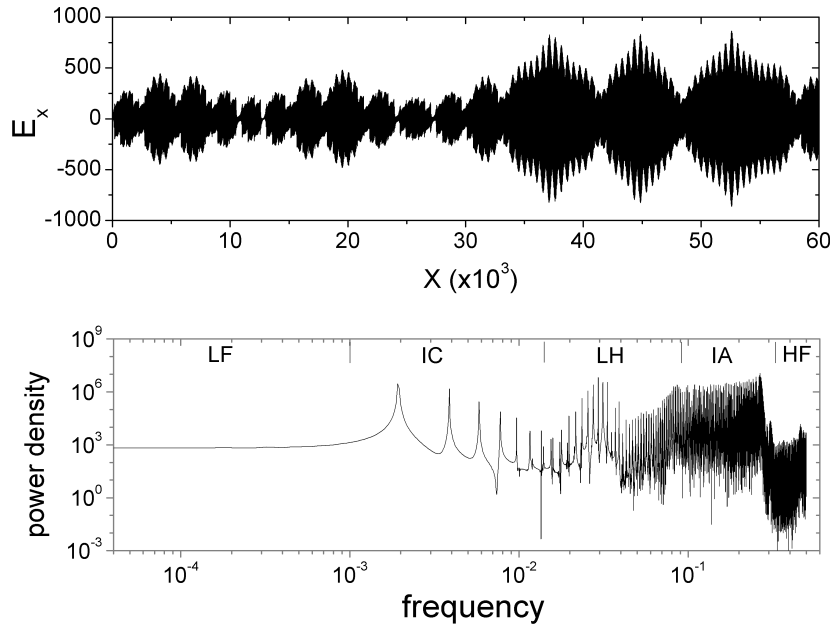

Fig. 18. A preliminary simulation to observed $\mathrm{LH}$ oscillitons under $U_{0}=(0.2,0,0), E_{0}=(0.5,0.5,1)$, and $B_{0}=(0.2,0.5,3)$ with $M=1$, $m_{\mathrm{i}} / m_{\mathrm{p}}=16, T_{\mathrm{e}} / T_{\mathrm{i}}=10$, and $v_{\mathrm{T}_{\mathrm{e}}} / c=0.05$. See Fig. 1 for a reference.

However, we would like to point out that higher-frequency oscillations should be measured if the payload resolution is high enough due to their modulations to the amplitude of $\mathrm{LH}$ packets.

Under initial conditions $U_{0}=(0.2,0,0), E_{0}=(0.5,0.5,1)$, and $B_{0}=(0.2,0.5,3)$, along with following input parameters $M=1, m_{\mathrm{i}} / m_{\mathrm{p}}=16, T_{\mathrm{e}} / T_{\mathrm{i}}=10$, and $v_{\mathrm{T}_{\mathrm{e}}} / c=0.05$, we calculate the LH-oscilliton wavefroms, as given by Fig. 18 where the electric wavefield component $E_{\mathrm{x}}$ (upper panel) and its spectrum (lower panel) are provided. The $E_{\mathrm{x}}$ panel conveys following messages about the oscillitons. Firstly, the series of packets has a dominant IC period of about $500 \lambda_{\text {De }}$, providing a frequency peak at $\sim 0.002 \omega_{\mathrm{pi}}$. Secondly, the envelop is modulated by LH oscillations of a period about $30 \lambda_{\mathrm{De}}$, offering a frequency of $\sim 0.03 \omega_{\mathrm{pi}}$. Lastly, superimposed upon the LH mode, there are higher-frequency IA oscillations of periods about several $\lambda_{\text {De }}$, contributing to a frequency band of tenths of $\omega_{\mathrm{pi}}$. These results are in agreement with the FFT spectrum: in the IC band, there are a series of IC harmonics with a fundamental peak of $0.0019 \omega_{\mathrm{pi}}$; in the LH band, there are LH peaks the dominant one of which is at $0.0315 \omega_{\mathrm{pi}}$; In the IA band, there is a narrow band up to $0.3 \omega_{\mathrm{pi}}$ the lowfrequency end of extends into the LH band. Above the IA band, the high-frequency noises are obvious in the HF region.

Though from Fig. 1, it is hard to identify the periodic feature of waves before 02:05:25.7, the upper panel in Fig. 18 reveals the seemingly periodic nature before $X=30 \times 10^{3}$. It is not hard to see two packets from $X=0$ to $X=25 \times 10^{3}$, each of which contains 5 small oscillitons with a period of $X=3000$. The period is about $X=12.5 \times 10^{3}$, the same as that of the three big oscillitons after $X=30 \times 10^{3}$. This very long period $\left(X=12.5 \times 10^{3}\right)$, along with the period of $X=3000$, should provide low-frequency (LF) harmonic peaks at about 
$8 \times 10^{-5}$ and $3.3 \times 10^{-4}$, respectively, in the FFT panel. Fortunately, we can discern a peak corresponding to the second one in the LF region, though it gives only a tiny hump in power. Unfortunately, the first one is unable to be trace out. This is understandable by realizing that there are 12 envelops of $X=3000$ from $X=0$ to $X=30 \times 10^{3}$, however only 3-5 $(X=12500)$-ones from $X=0$ to $X=60 \times 10^{3}$. The two lowerfrequency modulations than that of the IC mode may be aroused by some other mechanism irrelevant of the Landau damping effect (or, the kinetic resonance). (This is because in the present model, this effect is negligible $\left(\lambda_{\mathrm{FLR}} \ll 1\right)$ for any species, and the electromagnetic perturbation approach due to the resonance does not fit the present study; Kourakis and Shukla, 2004; Hirose, 2005, 2007.)

\section{Summary and discussion}

Kourakis and Shukla (2005) provided a generic methodological formulation for observed oscillitons which are featured by quickly-varying oscillations superimposed upon slowlyvarying solitary waves. Inspired by Sauer et al. (2003)'s study in a dusty plasma case where an addition of a second ion population in a single-ion plasma leads to significant modifications of solitary waves, we specialize Kourakis and Shukla (2005) model to explain the formation of abundantly observed LH-oscillitons in space plasmas.

Owing to the fact that the Landau damping is negligible $\left(\lambda_{\mathrm{FLR}} \ll 1\right)$ for any species, we employed a collision-free, two-fluid model to perform parameterized simulations. We started from exhibiting the excitation of the IA/IC solitary waves, and investigated the modulation of the IC/IA envelops by the electron inertia. The inertia triggers LH oscillitons characterized by a normal IC-period solitary envelop embedded by LH oscillations which contain higher-frequency but smaller-amplitude IA constituents. We exhibited the impact of the electron inertia on oscilliton packets via several input parameters like the Mach number, the electron-ion mass ratio, temperature ratio, etc. Unexpectedly, there exists a lower-frequency mode beyond the IC band. It is hard to be explained by the present hydrodynamic model. We will pay attention to it in our future work.

Though it has already been illustrated that the electron inertia behaves as one key to trigger LH oscillitons which are influenced by input parameters, we realize that initial conditions should also have impacts on the existence of oscillitons. Recall the first study of solitary waves by John Scott Russell: the speed of a ship triggers water solitary waves (e.g., Craik, 2004). Naturally, we are going to report in another paper the roles played by the initial conditions. Besides, we are very interested in the new Cluster observations on coherent whistler emissions in the magnetosphere (e.g., Dubinin et al., 2007). The measurements provided clear evidence that the magnetic field components are so perturbed as to form a sequence of oscilliton packets while the periodic structure was also revealed from the wavelet analysis (see Fig. 6 of that article). This will energize us to generalize Sauer et al. (2002)'s oblique-whistler model to warm-plasma cases in which particles are coupled with each other more closely via the pressure term.

Last but not least, we will try to find a link between the solitary waves and the two important categories of observations: transverse ion heating and broadband noise excitation. This subject is important. On one hand, it will provide a mechanism for the transverse ion heating via the nonMaxwellian velocity distributions brought about by space charges in geospace. On the other hand, it will serve as a reference in studying the relationship among nonlinear Alfvén waves, discontinuities, proton perpendicular acceleration, and magnetic holes/decreases in interplanetary space and the planetary magnetospheres (see new theories and observations by, e.g., Tsurutani et al., 2002a, b, 2005). The study will be dominantly based on two facts: (1) soliton trains carry electric fields contributed by space charges the density of which is periodic in both space and time for a single oscilliton train, as depicted in this paper; (2) the spatial extent of a single train perpendicular to the propagation direction is in scales of 2-20 ion Larmor radii (see e.g. Ergun, 1999) and there appears existing sets of trains propagating in background magnetic fields. A simple picture is as follows: ions (not those constituting solitary waves but surrounding ones in the vicinity, at least in the boundary layer, of the waves) are accelerated by a stochastic space-charge electric field, in both amplitude and time, contributed by all solitary trains in the perpendicular plane of the propagation direction. At any moment, If the field can be considered as proportional to the radius in a cylindrical geometry if the total space charges offered by solitary waves are uniform instantly, the $\boldsymbol{E} \times \boldsymbol{B}$ drift can drive ambient Maxwellian ions to non-Maxwellians transversely with observable orders of upgraded magnitude in temperature (Ma et al., 2009).

Luckily, this picture appears to be valid in view of observations (e.g., Ergun et al., 1998; Ergun, 1999; Pickett et al., 2005): magnetic flux tubes are always teeming with a dense cluster of soliton trains. Though solitary packets in each of the trains can be considered identical, different trains carry different packets with respective amplitudes, lifetimes, and scales. These divergences are originated from the saturated growths of linearization constrained by the input parameters and boundary conditions at specific positions and time. Undoubtedly, at any time, the collective behavior of the cluster of these solitary waves differs from that of a single solitary train: both the amplitude and the lifetime of space-charge density $n_{\mathrm{sc}}$ is random. In view of the measurements that solitary trains fill up all spaces in a magnetic flux tube, the whole tube cylinder has a space-charge density, $\tilde{n}_{\mathrm{c}}$, which is instantaneously uniform in space, at least to the leading order for simplicity, but stochastic in time. Here, the "uniform" denotes an average of space-charge densities carried by packets on different trains with different $n_{\mathrm{sc}}$, while "stochastic" 
expresses the random features of $n_{\mathrm{sc}}$ in both amplitude and lifetime. By employing such a physical model, a companion paper will introduce the study on the frequency sweeping of IC oscillations, an important subdivision of broadband noises.

It deserves to verify an essential condition in discussing electrostatic waves in this paper: the negligence of the Landau damping effect. This effect is very important for ion acoustic waves (see, e.g., Gary, 1993). It can only be neglected in cases, as mentioned in the beginning of Sect. 2, where the phase speed $\left(v_{\mathrm{p}}\right)$ of the waves is very large compared to the ion thermal speed $\left(v_{\mathrm{T}_{\mathrm{i}}}\right)$, and very small compared to the electron thermal speed $\left(v_{T_{\mathrm{e}}}\right)$, as expressed by Eq. (2). We show that this condition is satisfied automatically in employing the two-fluid model (e.g., Chapter 4, Bellan, 2008): by recasting the generalized dispersion relation, Eq. (B3), we have

$\omega^{2}=\frac{k^{2} c_{\mathrm{s}}^{2}}{1+\lambda_{\mathrm{De}}^{2} k^{2}}+\gamma k^{2} v_{\mathrm{T}_{\mathrm{i}}}^{2}$

which reproduces Eq. (4.38) in Bellan (2008). Because the wavelength $1 / k$ is tens of $\lambda_{\text {De }}$ (see, e.g., Fig. 4 ), we have $\lambda_{\mathrm{De}}^{2} k^{2} \ll 1$. Thus, Eq. (18) provides

$v_{\mathrm{T}_{\mathrm{i}}} \ll v_{\mathrm{p}} \sim \sqrt{c_{\mathrm{s}}^{2}+\gamma v_{\mathrm{T}_{\mathrm{i}}}^{2}} \ll v_{\mathrm{T}_{\mathrm{e}}}$

due to the fact that $m_{\mathrm{i}} \gg m_{\mathrm{e}}$ and $c_{\mathrm{s}} \gg 0$.

Notice that this requirement is not only applicable to regions where $\xi_{\mathrm{T}}=T_{\mathrm{e} 0} / T_{\mathrm{i} 0}>1$ (or, equivalently, $T_{\mathrm{e} 0}>T_{\mathrm{i} 0}$ ), but also to those where $T_{\mathrm{e} 0}<T_{\mathrm{i} 0}$. Table 1 lists some plasma parameters in auroral regions where $T_{\mathrm{e} 0}>T_{\mathrm{i} 0}$, calculated from measurements by the GEODESIC rocket (Burchill et al., 2004), the Freja satellite (Eriksson et al., 1994), and the FAST satellite (Ergun, 1999), respectively. By contrast, in most magnetosphic plasmas (especially encountering some extreme situations like, e.g., substorms), $\xi_{\mathrm{T}}$ lies between 1/12 and $1 / 3$, as reported by, e.g., AMPTE/IRM and Cluster observations in the Earth's central plasma sheet, the sheet boundary layers, and magnetosheath (Baumjohann, 1993; Phan et al., 1994; Lavraud, 2009). It is another interesting topic on the excitation of oscillitons under the condition of $\xi_{\mathrm{T}}<1$, besides the case introduced in this paper with $\xi_{\mathrm{T}}>1$.

To finalize this paper, we would like to point out that the descriptions of nonlinear plasma physics often suffer from a lack of a coherent nomenclature for the phenomena: one author's "electrostatic shock" is another authors "soliton". This fact was highlighted in the introduction to $\mathrm{Ma}$ and $\mathrm{Hi}$ rose (2009a) where the authors gave a thorough discussion of prior literature in this field, and showed that there are often differences in nomenclature used by different authors.
Table 1. Some typical plasma parameters in auroral plasmas measured by the Geodesic rocket (Burchill et al., 2004), the Freja satellite (Eriksson et al., 1994), and the FAST satellite (Ergun, 1999).

\begin{tabular}{lccc}
\hline Vehicle & Geodesic & Freja & FAST \\
\hline$m_{\mathrm{i}} / m_{\mathrm{p}}$ & 16 & $16 / 1$ & 1 \\
$B(\mathrm{mG})$ & 500 & 250 & 100 \\
$n_{0}\left(\mathrm{~cm}^{-3}\right)$ & $10^{6}$ & $10^{3}$ & 10 \\
$\Omega_{\mathrm{i}}(\mathrm{krad} / \mathrm{s})$ & 0.3 & $0.15 / 2.4$ & 1 \\
$\Omega_{\mathrm{e}}(\mathrm{krad} / \mathrm{s})$ & 8800 & 4400 & 1840 \\
$\omega_{\mathrm{LH}}(\mathrm{krad} / \mathrm{s})$ & 50 & $9 / 37$ & 4.14 \\
$T_{\mathrm{i}}(\mathrm{eV})$ & 0.1 & 0.2 & 300 \\
$T_{\mathrm{e}}(\mathrm{eV})$ & 0.2 & 0.3 & 700 \\
$v_{\mathrm{T}}(\mathrm{km} / \mathrm{s})$ & 0.77 & $1.10 / 4.38$ & 170 \\
$v_{\mathrm{T}}(\mathrm{km} / \mathrm{s})$ & 187 & 230 & $1.1 \times 10^{4}$ \\
$c_{\mathrm{S}}(\mathrm{km} / \mathrm{s})$ & 1 & $1.3 / 5.5$ & 260 \\
$c_{\mathrm{S}} B(\mathrm{mV} / \mathrm{m})$ & 50 & $33 / 140$ & 2600 \\
$\xi_{\mathrm{T}}$ & 2 & 1.5 & 2.3 \\
$\xi_{\mathrm{v}}$ & $6.2 \times 10^{-4}$ & $7.7 \times 10^{-4}$ & 0.04 \\
$\lambda_{\mathrm{De}}(\mathrm{m})$ & 0.003 & $0.13 / 0.14$ & 62 \\
\hline
\end{tabular}

\section{Appendix A}

\section{Dispersion relation of electrostatic IA waves}

Choosing $\boldsymbol{B}_{\mathrm{ext}}=B_{\mathrm{x} 0} \hat{\boldsymbol{e}}_{\mathrm{x}}, \boldsymbol{u}_{1}=u_{\mathrm{x} 1} \hat{\mathrm{e}}_{\mathrm{x}}, \boldsymbol{E}_{1}=E_{1 \mathrm{x}} \hat{\mathrm{e}}_{\mathrm{x}}, \boldsymbol{B}_{1}=0$, and $\boldsymbol{k}=k_{\mathrm{x}} \hat{\mathrm{e}}_{\mathrm{x}}$, while other components of perturbed parameters are neglected, the linearized set of Eq. (3) is as follows:

$$
\left.\begin{array}{l}
\frac{n_{\mathrm{e} 1}}{n_{0}}=i \frac{\Omega_{\mathrm{e}}}{B_{0}} \frac{k_{\mathrm{x}} E_{1 \mathrm{x}}}{\left(k_{\mathrm{x}} v_{\mathrm{T}}\right)^{2}} \\
\frac{n_{\mathrm{i} 1}}{n_{0}}=i \frac{\Omega_{\mathrm{i}}}{B_{0}} \frac{k_{\mathrm{x}} E_{1 \mathrm{x}}}{\omega^{2}-\left(\gamma k_{\mathrm{x}} v_{\mathrm{T}_{\mathrm{i}}}\right)^{2}} \\
k_{\mathrm{x}} E_{1 \mathrm{x}}=-i \frac{e n_{0}}{\epsilon_{0}}\left(\frac{n_{\mathrm{i} 1}}{n_{0}}-\frac{n_{\mathrm{e} 1}}{n_{0}}\right)
\end{array}\right\}
$$

by neglecting the electron inertia due to its small mass relative to that of ions. Let $E_{1 \mathrm{x}}=-i k_{\mathrm{x}} \varphi$. We have

$$
\left.\begin{array}{l}
\frac{n_{\mathrm{e} 1}}{n_{0}}=\frac{\Omega_{\mathrm{e}}}{B_{0}} \frac{\varphi}{v_{\mathrm{T}_{\mathrm{e}}}^{2}} \\
\frac{n_{\mathrm{i} 1}}{n_{0}}=k_{\mathrm{x}}^{2} \frac{\Omega_{\mathrm{i}}}{B_{0}} \frac{\varphi}{\omega^{2}-\gamma v_{\mathrm{T}_{\mathrm{i}}}^{2} k_{\mathrm{x}}^{2}} \\
\varphi=\frac{\mathrm{en}_{0}}{\epsilon_{0} k_{\mathrm{x}}^{2}}\left(\frac{n_{\mathrm{i} 1}}{n_{0}}-\frac{n_{\mathrm{e} 1}}{n_{0}}\right)
\end{array}\right\}
$$

which leads a dispersion relation of the IA waves:

$$
\omega^{2}=\gamma v_{\mathrm{T}_{\mathrm{i}}}^{2} k_{\mathrm{x}}^{2}+\frac{\omega_{\mathrm{pi}}^{2}}{1+\left(\frac{k_{\mathrm{De}}}{k_{\mathrm{x}}}\right)^{2}}
$$

where $k_{\mathrm{De}}=\lambda \lambda_{\mathrm{De}}^{-1}=\omega_{\mathrm{pe}} / v_{\mathrm{T}_{\mathrm{e}}}$ is the Debye wavelength. Because $k_{\mathrm{De}} \gg k_{\mathrm{X}}$, the dispersion relation becomes

$\omega^{2}=\left(\gamma v_{\mathrm{T}_{\mathrm{i}}}^{2}+c_{\mathrm{S}}^{2}\right) k_{\mathrm{x}}^{2}$. 


\section{Appendix B}

\section{Dispersion relation of electrostatic IA/IC waves}

Using $\boldsymbol{b}=\boldsymbol{B}_{\text {ext }} /\left|\boldsymbol{B}_{\text {ext }}\right|$, the linearized set of Eq. (3) is as follows:

$$
\left.\begin{array}{l}
\frac{n_{\mathrm{e} 1}}{n_{0}}=-i \frac{\Omega_{\mathrm{e}}}{\omega^{2}-v_{\mathrm{T}_{\mathrm{e}}}^{2} k^{2}}\left[\frac{\boldsymbol{k} \cdot \boldsymbol{E}_{1}}{B_{0}}+\boldsymbol{u}_{\mathrm{e} 1} \cdot(\boldsymbol{b} \times \boldsymbol{k})\right] \\
\frac{n_{\mathrm{i} 1}}{n_{0}}=i \frac{\Omega_{\mathrm{i}}}{\omega^{2}-\gamma v_{\mathrm{T}_{\mathrm{i}}}^{2} k^{2}}\left[\frac{\boldsymbol{k} \cdot \boldsymbol{E}_{1}}{B_{0}}+\boldsymbol{u}_{\mathrm{i} 1} \cdot(\boldsymbol{b} \times \boldsymbol{k})\right] \\
\boldsymbol{k} \times \boldsymbol{E}_{1}=\omega \boldsymbol{B}_{1} \\
i \boldsymbol{k} \times \boldsymbol{B}_{1}=\mu_{0} n_{0} e\left(\boldsymbol{u}_{\mathrm{i} 1}-\boldsymbol{u}_{\mathrm{e} 1}\right) \\
\boldsymbol{k} \cdot \boldsymbol{E}_{1}=-i \frac{\mathrm{e} \mathrm{e}_{0}}{\epsilon_{0}}\left(\frac{n_{\mathrm{i} 1}}{n_{0}}-\frac{n_{\mathrm{e} 1}}{n_{0}}\right) \\
\boldsymbol{k} \cdot \boldsymbol{B}_{1}=0
\end{array}\right\}
$$

Neglecting electron inertia under electrostatic case, $\boldsymbol{B}_{1}=0$, we have

$$
\left.\begin{array}{l}
\frac{n_{\mathrm{i} 1}}{n_{0}}=\frac{n_{\mathrm{e} 1}}{n_{0}} \frac{v_{\mathrm{T}_{\mathrm{e}}}^{2} k^{2}}{\Omega_{\mathrm{e}}} \frac{\Omega_{\mathrm{i}}}{\omega^{2}-\gamma v_{\mathrm{T}_{\mathrm{i}}}^{2} k^{2}} \\
\boldsymbol{k} \cdot \boldsymbol{E}_{1}=-i \frac{\mathrm{en}_{0}}{\epsilon_{0}}\left(\frac{n_{\mathrm{i} 1}}{n_{0}}-\frac{n_{\mathrm{e} 1}}{n_{0}}\right)
\end{array}\right\}
$$

Using $\boldsymbol{E}_{1}=-i \boldsymbol{k} \varphi$, and $n_{\mathrm{e} 1} / n_{0} \approx e \varphi /\left(k_{\mathrm{B}} T_{\mathrm{e}}\right)$, we have

$$
\omega^{2}=\gamma v_{\mathrm{T}_{\mathrm{i}}}^{2} k^{2}+\frac{\omega_{\mathrm{pi}}^{2}}{1+\left(\frac{k_{\mathrm{De}}}{k}\right)^{2}}
$$

which produces

$$
\omega^{2}=\left(\gamma v_{\mathrm{T}_{\mathrm{i}}}^{2}+c_{\mathrm{s}}^{2}\right) k^{2}
$$

for $k_{\mathrm{De}} \gg k$. Clearly, Eq. (B3) is the generalization of the specialized IA dispersion relation, Eq. (A4).

In addition to the propagating IA waves, there are local non-propagating IC oscillations in the plane perpendicular to the propagating direction of the IA modes. In the slabmodel case as introduced in Sect. 2 where parameters are only dependent of $x$, we obtain the linearized equations of Eq. (3) for $\boldsymbol{u}_{\mathrm{i} 1 \perp}=\left\{u_{\mathrm{i} 1 \mathrm{y}}, u_{\mathrm{i} 1 \mathrm{z}}\right\}$ of ions as follows:

$$
\left.\begin{array}{l}
i \omega u_{\mathrm{i} 1 \mathrm{y}}=-\Omega_{\mathrm{i}} u_{\mathrm{i} 1 \mathrm{z}} \\
i \omega u_{\mathrm{i} 1 \mathrm{z}}=\Omega_{\mathrm{i}} u_{\mathrm{i} 1 \mathrm{y}}
\end{array}\right\}
$$

which leads to

$\omega=\Omega_{\mathrm{i}}$

Clearly, this is an ion cyclotron oscillation in the plane perpendicular to $\hat{\boldsymbol{e}}_{\mathrm{x}}$. Note that the oscillation frequency is not an invariable if the strength of the external magnetic field changes in space and/or time which is excited by nonlinear waves.

\section{Appendix C}

\section{Dispersion relation of generalized LH waves}

For convenience, we temporally transform coordinates $\left\{\hat{\boldsymbol{e}}_{\mathrm{x}}, \hat{\boldsymbol{e}}_{\mathrm{y}}, \hat{\boldsymbol{e}}_{\mathrm{z}}\right\}$ to $\left\{\hat{\boldsymbol{e}}_{\mathrm{x}}^{\prime}, \hat{\boldsymbol{e}}_{\mathrm{y}}^{\prime}, \boldsymbol{b}\right\}$ in which $\boldsymbol{b}=\boldsymbol{B}_{\mathrm{ext}} /\left|\boldsymbol{B}_{\mathrm{ext}}\right|$ with $\left|\boldsymbol{B}_{\text {ext }}\right|=\sqrt{B_{\mathrm{x} 0}^{2}+B_{\mathrm{y} 0}^{2}+B_{\mathrm{z} 0}^{2}}=B_{\mathrm{z} 0}^{\prime}$. By using $\omega$ and $k$ to represent the wave frequency and the amplitude of the wave vector $\boldsymbol{k}=k_{\mathrm{x}} \hat{\boldsymbol{e}}_{\mathrm{x}}^{\prime}+k_{\mathrm{y}} \hat{\boldsymbol{e}}_{\mathrm{y}}^{\prime}+k_{\mathrm{z}} \boldsymbol{b}$ (with $k^{2}=k_{\perp}^{2}+k_{\mathrm{z}}^{2}$ in which $k_{\perp}^{2}=k_{\mathrm{x}}^{2}+k_{\mathrm{y}}^{2}$ ), respectively, we obtain a linearized set of equations from Eq. (3) as follows:

$$
\left.\begin{array}{l}
\omega\left(\frac{n_{\mathrm{e} 1}}{n_{0}}\right)=\boldsymbol{k} \cdot \boldsymbol{u}_{\mathrm{e} 1} \\
\omega\left(\frac{n_{\mathrm{i} 1}}{n_{0}}\right)=\boldsymbol{k} \cdot \boldsymbol{u}_{\mathrm{i} 1} \\
i \omega \boldsymbol{u}_{\mathrm{e} 1}=v_{\mathrm{T}_{\mathrm{e}}}^{2} i \boldsymbol{k}\left(\frac{n_{\mathrm{e} 1}}{n_{0}}\right)+\Omega_{\mathrm{e}}\left(\frac{\boldsymbol{E}_{1}}{B_{0}}+\boldsymbol{u}_{\mathrm{e} 1} \times \boldsymbol{b}\right) \\
i \omega \boldsymbol{u}_{\mathrm{i} 1}=\gamma v_{\mathrm{T}_{\mathrm{i}}}^{2} i \boldsymbol{k}\left(\frac{n_{\mathrm{i} 1}}{n_{0}}\right)-\Omega_{\mathrm{i}}\left(\frac{\boldsymbol{E}_{1}}{B_{0}}+\boldsymbol{u}_{\mathrm{i} 1} \times \boldsymbol{b}\right) \\
\boldsymbol{k} \times \boldsymbol{E}_{1}=\omega \boldsymbol{B}_{1} \\
i \boldsymbol{k} \times \boldsymbol{B}_{1}=\mu_{0} n_{0} e\left(\boldsymbol{u}_{\mathrm{i} 1}-\boldsymbol{u}_{\mathrm{e} 1}\right) \\
i \boldsymbol{k} \cdot \boldsymbol{E}_{1}=\frac{\mathrm{en}}{\epsilon_{0}}\left(\frac{n_{\mathrm{i} 1}}{n_{0}}-\frac{n_{\mathrm{e} 1}}{n_{0}}\right) \\
i \boldsymbol{k} \cdot \boldsymbol{B}_{1}=0
\end{array}\right\}
$$

where a homogeneous plasma is assumed with $\boldsymbol{u}_{0}=0$. The two momentum equations give

$$
\left.\begin{array}{l}
i \omega \boldsymbol{k} \cdot \boldsymbol{u}_{\mathrm{e} 1}=v_{\mathrm{T}_{\mathrm{e}}}^{2} i k^{2}\left(\frac{n_{\mathrm{e} 1}}{n_{0}}\right)+\Omega_{\mathrm{e}}\left(\frac{\boldsymbol{k} \cdot \boldsymbol{E}_{1}}{B_{0}}+\boldsymbol{k} \cdot\left(\boldsymbol{u}_{\mathrm{e} 1} \times \boldsymbol{b}\right)\right) \\
i \omega \boldsymbol{k} \cdot \boldsymbol{u}_{\mathrm{i} 1}=\gamma v_{\mathrm{T}_{\mathrm{i}}}^{2} i k^{2}\left(\frac{n_{\mathrm{i} 1}}{n_{0}}\right)-\Omega_{\mathrm{i}}\left(\frac{\boldsymbol{k} \cdot \boldsymbol{E}_{1}}{B_{0}}+\boldsymbol{k} \cdot\left(\boldsymbol{u}_{\mathrm{i} 1} \times \boldsymbol{b}\right)\right)
\end{array}\right\}
$$

which leads to

$$
\left.\begin{array}{l}
\omega^{2}\left(\frac{n_{\mathrm{e} 1}}{n_{0}}\right)=v_{\mathrm{T}_{\mathrm{e}}}^{2} k^{2}\left(\frac{n_{\mathrm{e} 1}}{n_{0}}\right)-i \Omega_{\mathrm{e}}\left(\frac{\boldsymbol{k} \cdot \boldsymbol{E}_{1}}{B_{0}}+\boldsymbol{k} \cdot\left(\boldsymbol{u}_{\mathrm{e} 1} \times \boldsymbol{b}\right)\right) \\
\omega^{2}\left(\frac{n_{\mathrm{i} 1}}{n_{0}}\right)=\gamma v_{\mathrm{T}_{\mathrm{i}}}^{2} k^{2}\left(\frac{n_{\mathrm{i} 1}}{n_{0}}\right)+i \Omega_{\mathrm{i}}\left(\frac{\boldsymbol{k} \cdot \boldsymbol{E}_{1}}{B_{0}}+\boldsymbol{k} \cdot\left(\boldsymbol{u}_{\mathrm{i} 1} \times \boldsymbol{b}\right)\right)
\end{array}\right\}
$$

by employing the two density equations in Eq. (C1). Together with the electromagnetic equations, this equation produces

$$
\left.\begin{array}{l}
\frac{\mu_{0} n_{0} e}{\Omega_{\mathrm{e}}}\left(\omega^{2}-v_{\mathrm{T}_{\mathrm{e}}}^{2} k^{2}\right)\left(\frac{n_{\mathrm{e} 1}}{n_{0}}\right)+\frac{\mu_{0} n_{0} e}{\Omega_{\mathrm{i}}}\left(\omega^{2}-\gamma v_{\mathrm{T}_{\mathrm{i}}}^{2} k^{2}\right)\left(\frac{n_{\mathrm{i} 1}}{n_{0}}\right) \\
=k^{2} \frac{k_{\mathrm{x}} E_{1 \mathrm{y}}-k_{\mathrm{y}} E_{1 \mathrm{x}}}{\omega}=k^{2} \boldsymbol{B}_{1} \cdot \boldsymbol{b}
\end{array}\right\}
$$

in which $n_{\mathrm{e} 1}, n_{\mathrm{i} 1}$, and $E_{\mathrm{x}}, E_{\mathrm{y}}$ can be obtained by solving the two momentum equations in Eq. (C1). For electrons, we have

$\left[\begin{array}{lll}a_{11} & a_{12} & a_{13} \\ a_{21} & a_{22} & a_{23} \\ a_{31} & a_{32} & a_{33}\end{array}\right]\left[\begin{array}{c}u_{\mathrm{e} 1 \mathrm{x}} \\ u_{\mathrm{e} 1 \mathrm{y}} \\ u_{\mathrm{e} 1 \mathrm{z}}\end{array}\right]=i \frac{\Omega_{\mathrm{e}}}{\omega B_{0}}\left[\begin{array}{c}E_{1 \mathrm{x}} \\ E_{1 \mathrm{y}} \\ E_{1 \mathrm{z}}\end{array}\right]$ 
in which

$$
\begin{aligned}
& a_{11}=-\left(1-\frac{v_{\mathrm{T}_{\mathrm{e}}}^{2} k_{\mathrm{x}}^{2}}{\omega^{2}}\right) \\
& a_{12}=-\left(i \frac{\Omega_{\mathrm{e}}}{\omega}-\frac{v_{\mathrm{T}_{\mathrm{e}}}^{2} k_{\mathrm{x}} k_{\mathrm{y}}}{\omega^{2}}\right) \\
& a_{13}=\frac{v_{\mathrm{T}_{\mathrm{e}}}^{2} k_{\mathrm{x}} k_{\mathrm{z}}}{\omega^{2}} \\
& a_{21}=i \frac{\Omega_{\mathrm{e}}}{\omega}+\frac{v_{\mathrm{T}_{\mathrm{e}}}^{2} k_{\mathrm{x}} k_{\mathrm{y}}}{\omega^{2}} \\
& a_{22}=-\left(1-\frac{v_{\mathrm{T}_{\mathrm{e}}}^{2} k_{\mathrm{y}}^{2}}{\omega^{2}}\right) \\
& a_{23}=\frac{v_{\mathrm{T}_{\mathrm{e}}}^{2} k_{\mathrm{y}} k_{\mathrm{z}}}{\omega^{2}} \\
& a_{31}=\frac{v_{\mathrm{T}_{\mathrm{e}}}^{2} k_{\mathrm{x}} k_{\mathrm{z}}}{\omega^{2}} \\
& a_{32}=\frac{v_{\mathrm{T}_{\mathrm{e}}}^{2} k_{\mathrm{y}} k_{\mathrm{z}}}{\omega^{2}} \\
& a_{33}=-\left(1-\frac{v_{\mathrm{T}_{\mathrm{e}}}^{2} k_{\mathrm{z}}^{2}}{\omega^{2}}\right)
\end{aligned}
$$

The solution is:

$u_{\mathrm{e} 1 \mathrm{x}}=\frac{\Delta_{1}}{\Delta}, u_{\mathrm{e} 1 \mathrm{y}}=\frac{\Delta_{2}}{\Delta}, u_{\mathrm{e} 1 \mathrm{z}}=\frac{\Delta_{3}}{\Delta}$

where

$$
\begin{aligned}
& \Delta=\left|\begin{array}{lll}
a_{11} & a_{12} & a_{13} \\
a_{21} & a_{22} & a_{23} \\
a_{31} & a_{32} & a_{33}
\end{array}\right|=\frac{v_{\mathrm{T}_{\mathrm{e}}}^{2} k_{\perp}^{2}}{\omega^{2}}+\left(1-\frac{\Omega_{\mathrm{e}}^{2}}{\omega^{2}}\right)\left(\frac{v_{\mathrm{T}_{\mathrm{e}}}^{2} k_{\mathrm{z}}^{2}}{\omega^{2}}-1\right) \\
& \Delta_{1}=i \frac{\Omega_{\mathrm{e}}}{\omega B_{0}}\left|\begin{array}{ccc}
E_{1 \mathrm{x}} & a_{12} & a_{13} \\
E_{1 \mathrm{y}} & a_{22} & a_{23} \\
E_{1 \mathrm{z}} & a_{32} & a_{33}
\end{array}\right| \\
& =i \frac{\Omega_{\mathrm{e}}}{\omega}\left\{\left[1-\frac{v_{\mathrm{T}_{\mathrm{e}}}^{2}\left(k_{\mathrm{y}}^{2}+k_{\mathrm{z}}^{2}\right)}{\omega^{2}}\right] \frac{E_{1 \mathrm{x}}}{B_{0}}\right. \\
& +\left[\frac{v_{\mathrm{T}_{\mathrm{e}}}^{2}}{\omega^{2}}\left(k_{\mathrm{x}} k_{\mathrm{y}}+i \frac{\Omega_{\mathrm{e}}}{\omega} k_{\mathrm{z}}^{2}\right)-i \frac{\Omega_{\mathrm{e}}}{\omega}\right] \frac{E_{1 \mathrm{y}}}{B_{0}} \\
& \left.+\frac{v_{\mathrm{T}_{\mathrm{e}}}^{2} k_{\mathrm{z}}}{\omega^{2}}\left(k_{\mathrm{x}}-i \frac{\Omega_{\mathrm{e}}}{\omega} k_{\mathrm{y}}\right) \frac{E_{1 \mathrm{z}}}{B_{0}}\right\}
\end{aligned}
$$

$$
\begin{aligned}
& \Delta_{2}=i \frac{\Omega_{\mathrm{e}}}{\omega B_{0}}\left|\begin{array}{ccc}
a_{11} & E_{1 \mathrm{x}} & a_{13} \\
a_{21} & E_{1 \mathrm{y}} & a_{23} \\
a_{31} & E_{1 \mathrm{z}} & a_{33}
\end{array}\right| \\
& =i \frac{\Omega_{\mathrm{e}}}{\omega}\left\{\left[\begin{array}{ll}
\left.\frac{v_{\mathrm{T}}^{2}}{\omega^{2}}\left(k_{\mathrm{x}} k_{\mathrm{y}}-i \frac{\Omega_{\mathrm{e}}}{\omega} k_{\mathrm{z}}^{2}\right)+i \frac{\Omega_{\mathrm{e}}}{\omega}\right] \frac{E_{1 \mathrm{x}}}{B_{0}} \\
\left.+\left[\begin{array}{ll}
1-\frac{v_{\mathrm{T}_{\mathrm{e}}}^{2}}{\left.k_{\mathrm{x}}^{2}+k_{\mathrm{z}}^{2}\right)} \\
\omega^{2}
\end{array}\right] \frac{E_{1 \mathrm{y}}}{B_{0}}+\frac{v_{\mathrm{T}_{\mathrm{e}}}^{2} k_{\mathrm{z}}}{\omega^{2}}\left(k_{\mathrm{y}}+i \frac{\Omega_{\mathrm{e}}}{\omega} k_{\mathrm{x}}\right) \frac{E_{1 \mathrm{z}}}{B_{0}}\right\} \\
\Delta_{3}=i \frac{\Omega_{\mathrm{e}}}{\omega B_{0}}\left|\begin{array}{lll}
a_{11} & a_{12} & E_{1 \mathrm{x}} \\
a_{21} & a_{22} & E_{1 \mathrm{y}} \\
a_{31} & a_{32} & E_{1 \mathrm{z}}
\end{array}\right| \\
=i \frac{\Omega_{\mathrm{e}}}{\omega}\left[\frac{v_{\mathrm{T}_{\mathrm{e}}}^{2} k_{\mathrm{z}}}{\omega^{2}}\left(k_{\mathrm{x}}+i \frac{\Omega_{\mathrm{e}}}{\omega} k_{\mathrm{y}}\right) \frac{E_{1 \mathrm{x}}}{B_{0}}\right. \\
\left.+\frac{v_{\mathrm{T}_{\mathrm{e}}}^{2} k_{\mathrm{z}}}{\omega^{2}}\left(k_{\mathrm{y}}-i \frac{\Omega_{\mathrm{e}}}{\omega} k_{\mathrm{x}}\right) \frac{E_{1 \mathrm{y}}}{B_{0}}+\left(1-\frac{\Omega_{\mathrm{e}}^{2}}{\omega^{2}}-\frac{v_{\mathrm{T}_{\mathrm{e}}}^{2} k_{\perp}^{2}}{\omega^{2}}\right) \frac{E_{1 \mathrm{z}}}{B_{0}}\right]
\end{array}\right\}\right.
\end{aligned}
$$

Thus,

$$
\left.\begin{array}{l}
k_{\mathrm{x}} \Delta_{1}+k_{\mathrm{y}} \Delta_{2}+k_{\mathrm{z}} \Delta_{3}=i \frac{\Omega_{\mathrm{e}}}{\omega}\left[\left(k_{\mathrm{x}}+i \frac{\Omega_{\mathrm{e}}}{\omega} k_{\mathrm{y}}\right) \frac{E_{1 \mathrm{x}}}{B_{0}}\right. \\
\left.+\left(k_{\mathrm{y}}-i \frac{\Omega_{\mathrm{e}}}{\omega} k_{\mathrm{x}}\right) \frac{E_{1 y}}{B_{0}}+k_{\mathrm{z}}\left(1-\frac{\Omega_{\mathrm{e}}^{2}}{\omega^{2}}\right) \frac{E_{1 \mathrm{z}}}{B_{0}}\right]
\end{array}\right\}
$$

Then,

$$
\frac{n_{\mathrm{e} 1}}{n_{0}}=i \frac{\Omega_{\mathrm{e}}}{B_{0}} \frac{\boldsymbol{k} \cdot \boldsymbol{E}_{1}-\frac{\Omega_{\mathrm{e}}^{2}}{\omega^{2}} k_{\mathrm{z}} E_{1 \mathrm{z}}-i \Omega_{\mathrm{e}} \boldsymbol{B}_{1} \cdot \boldsymbol{b}}{k^{2} v_{\mathrm{T}_{\mathrm{e}}}^{2}\left[1-\frac{\Omega_{\mathrm{e}}^{2}}{\omega^{2}}\left(\frac{k_{\mathrm{z}}}{k}\right)^{2}\right]-\omega^{2}\left(1-\frac{\Omega_{\mathrm{e}}^{2}}{\omega^{2}}\right)}
$$

where $\omega \boldsymbol{B}_{1} \cdot \boldsymbol{b}=\left(\boldsymbol{k} \times \boldsymbol{E}_{1}\right) \cdot \boldsymbol{b}$ is used.

Similarly,

$$
\frac{n_{\mathrm{i} 1}}{n_{0}}=-i \frac{\Omega_{\mathrm{i}}}{B_{0}} \frac{\boldsymbol{k} \cdot \boldsymbol{E}_{1}-\frac{\Omega_{\mathrm{i}}^{2}}{\omega^{2}} k_{\mathrm{z}} E_{1 \mathrm{z}}+i \Omega_{\mathrm{i}} \boldsymbol{B}_{1} \cdot \boldsymbol{b}}{\frac{5}{3} v_{\mathrm{T}_{\mathrm{i}}}^{2}\left[1-\frac{\Omega_{\mathrm{i}}^{2}}{\omega^{2}}\left(\frac{k_{\mathrm{z}}}{k}\right)^{2}\right]-\omega^{2}\left(1-\frac{\Omega_{\mathrm{i}}^{2}}{\omega^{2}}\right)}
$$

Equation $(\mathrm{C} 4, \mathrm{C} 13$, and $\mathrm{C} 14)$, together with Poisson equation, produce

$$
\left.\begin{array}{c}
k_{\perp}^{2}\left[1-\frac{\omega_{\mathrm{pi}}^{2} \omega^{2}}{\left(\omega^{2}-\Omega_{\mathrm{i}}^{2}\right)\left(\omega^{2}-\frac{5}{3} k_{\mathrm{z}}^{2} v_{\mathrm{T}_{\mathrm{i}}}^{2}\right)-\frac{5}{3} \omega^{2} k_{\perp}^{2} v_{\mathrm{T}_{\mathrm{i}}}^{2}}\right. \\
\left.-\frac{\omega_{\mathrm{pe}}^{2} \omega^{2}}{\left(\omega^{2}-\Omega_{\mathrm{e}}^{2}\right)\left(\omega^{2}-k_{\mathrm{z}}^{2} v_{\mathrm{T}_{\mathrm{e}}}^{2}\right)-\omega^{2} k_{\perp}^{2} v_{\mathrm{T}_{\mathrm{e}}}^{2}}\right] \\
+k_{\mathrm{z}}^{2}\left[1-\frac{\omega_{\mathrm{pi}}^{2}\left(\omega^{2}-\Omega_{\mathrm{i}}^{2}-\frac{5}{3} k_{\perp}^{2} v_{\mathrm{T}_{\mathrm{i}}}^{2}\right)}{\left(\omega^{2}-\Omega_{\mathrm{i}}^{2}\right)\left(\omega^{2}-\frac{5}{3} k_{\mathrm{z}}^{2} v_{\mathrm{T}_{\mathrm{i}}}^{2}\right)-\frac{5}{3} \omega^{2} k_{\perp}^{2} v_{\mathrm{T}_{\mathrm{i}}}^{2}}\right. \\
\left.-\frac{\omega_{\mathrm{pe}}^{2}\left(\omega^{2}-\Omega_{\mathrm{e}}^{2}-k_{\perp}^{2} v_{\mathrm{T}_{\mathrm{e}}}^{2}\right)}{\left(\omega^{2}-\Omega_{\mathrm{e}}^{2}\right)\left(\omega^{2}-k_{\mathrm{z}}^{2} v_{\mathrm{T}_{\mathrm{e}}^{2}}^{2}\right)-\omega^{2} k_{\perp}^{2} v_{\mathrm{T}_{\mathrm{e}}}^{2}}\right]=0
\end{array}\right\}
$$


or, alternatively,

$$
\left.\begin{array}{c}
1=\left[\frac{\omega_{\mathrm{pi}}^{2}}{\left(\omega^{2}+\frac{5}{3} k^{2} v_{\mathrm{T}_{\mathrm{i}}}^{2} \frac{\Omega_{\mathrm{i}}^{2}}{\omega^{2}} \cos ^{2} \theta\right)-\omega_{\mathrm{i}}^{2}}\right. \\
\left.-\frac{\omega_{\mathrm{pe}}^{2}}{\omega_{\mathrm{e}}^{2}-\left(\omega^{2}+k^{2} v_{\mathrm{T}_{\mathrm{e}}}^{2} \frac{\Omega_{\mathrm{e}}^{2}}{\omega^{2}} \cos ^{2} \theta\right)}\right] \sin ^{2} \theta \\
+\left[\frac{\omega_{\mathrm{pi}}^{2}\left(1-\frac{\Omega_{\mathrm{i}}^{2}}{\omega^{2}}\right)}{\left(\omega^{2}+\frac{5}{3} k^{2} v_{\mathrm{T}_{\mathrm{i}}}^{2} \frac{\Omega_{\mathrm{i}}^{2}}{\omega^{2}} \cos ^{2} \theta\right)-\omega_{\mathrm{i}}^{2}}\right. \\
\left.-\frac{\omega_{\mathrm{pe}}^{2}\left(1-\frac{\Omega_{\mathrm{e}}^{2}}{\omega^{2}}\right)}{\omega_{\mathrm{e}}^{2}-\left(\omega^{2}+k^{2} v_{\mathrm{T}_{\mathrm{e}}}^{2} \frac{\Omega_{\mathrm{e}}^{2}}{\omega^{2}} \cos ^{2} \theta\right)}\right] \cos ^{2} \theta
\end{array}\right\}
$$

in the electrostatic case where $\boldsymbol{B}_{1}=0$, where $\cos \theta=k_{\mathrm{z}} / k$, $\sin \theta=k_{\perp} / k, \omega_{\mathrm{e}}=\sqrt{\Omega_{\mathrm{e}}^{2}+k^{2} v_{\mathrm{T}_{\mathrm{e}}}^{2}}$, and $\omega_{\mathrm{i}}=\sqrt{\Omega_{\mathrm{i}}^{2}+\gamma k^{2} v_{\mathrm{T}_{\mathrm{i}}}^{2}}$ are the pseudo-Bohm-Gross frequencies of electrons and ions, respectively. Notice that the electric displacement term is neglected due to $v_{\mathrm{p}}^{2} \ll c^{2}$ where $v_{\mathrm{p}}=\omega / k$ is the phase speed.

\section{C1 Modes along $b$}

In this case, $\theta=0^{\circ}$. Equation (C16) becomes

$$
1=\frac{\omega_{\mathrm{pi}}^{2}}{\omega^{2}-\gamma k^{2} v_{\mathrm{T}_{\mathrm{i}}}^{2}}-\frac{\omega_{\mathrm{pe}}^{2}}{k^{2} v_{\mathrm{T}_{\mathrm{e}}}^{2}-\omega^{2}}
$$

which exhibits several propagating modes under different cases related to $v_{T \alpha}$ within respective ranges of $v_{\mathrm{p}}$.

\section{C1.1 Both electron and ion inertia involved}

In this case, $v_{\mathrm{T}_{\mathrm{i}}} \ll v_{\mathrm{p}} \ll v_{\mathrm{T}_{\mathrm{e}}}$. Equation (C17) gives

$\omega^{2}=\frac{\left(k \lambda_{\text {De }} \omega_{\text {pi }}\right)^{2}}{\left(k \lambda_{\text {De }}\right)^{2}+1}$

which leads to, on one hand,

$\omega^{2}=\gamma_{\mathrm{e}}\left(k v_{\mathrm{T}_{\mathrm{e}}}\right)^{2}$

if $k \ll \lambda_{\mathrm{De}}^{-1}$; on the other hand,

$\omega=\omega_{\mathrm{pi}}$

if $k \gg \lambda \lambda_{\mathrm{De}}^{-1}$.

\section{C1.2 Electron inertia neglected}

In this case, $v_{\mathrm{p}} \sim v_{\mathrm{T}_{\mathrm{i}}}$. Equation (C17) gives

$\omega^{2}=\gamma k^{2} v_{\mathrm{T}_{\mathrm{i}}}^{2}+\omega_{\mathrm{pi}}^{2}$

\section{C1.3 Ion inertia neglected}

In this case, $v_{\mathrm{p}} \sim v_{\mathrm{T}_{\mathrm{e}}}$. Equation (C17) gives

$\omega^{2}=k^{2} v_{\mathrm{T}_{\mathrm{e}}}^{2}+\omega_{\mathrm{pe}}^{2}$

\section{C2 Modes in the plane perpendicular to $\mathrm{b}$}

In this case, $\theta=90^{\circ}$. Equation (C16) reduces to

$$
1=\frac{\omega_{\mathrm{pi}}^{2}}{\omega^{2}-\omega_{\mathrm{i}}^{2}}-\frac{\omega_{\mathrm{pe}}^{2}}{\omega_{\mathrm{e}}^{2}-\omega^{2}}
$$

which exhibits more complicated propagating modes through $\omega_{\alpha}$ under different conditions.

\section{C2.1 Both electron and ion inertia involved}

In this case, $\omega_{\mathrm{i}} \ll \omega \ll \omega_{\mathrm{e}}$. Equation (C23) gives the Lowerhybrid $(\mathrm{LH})$ mode $\left(\omega_{\mathrm{LH}}\right)$ :

$\omega^{2}=\frac{\omega_{\mathrm{pi}}^{2}}{1+\omega_{\mathrm{pe}}^{2} / \omega_{\mathrm{e}}^{2}}=\omega_{\mathrm{LH}}^{2}(k)$

which leads to, on one hand,

$\omega_{\mathrm{LH}}(k)=\sqrt{\omega_{\mathrm{i}} \omega_{\mathrm{e}}}$

in a weak magnetic field $\left(\omega_{\mathrm{e}} \ll \omega_{\text {pe }}\right.$ in, e.g., ionospheric plasmas); on the other hand,

$\omega_{\mathrm{LH}}=\omega_{\mathrm{pi}}$

in a strong magnetic field $\left(\omega_{\mathrm{e}} \gg \omega_{\text {pe }}\right.$ in, e.g., pulsar or blackhole plasmas).

\section{C2.2 Electron inertia neglected}

In this case, $\omega \sim \omega_{\mathrm{i}} \ll \omega_{\mathrm{e}}$. Equation (C23) gives the ion upperhybrid (iUH) mode $\left(\omega_{\mathrm{iUH}}\right)$ :

$\omega^{2}=\omega_{\mathrm{i}}^{2}+\omega_{\mathrm{pi}}^{2}=\omega_{\mathrm{iUH}}^{2}(k)$

which leads to the ion gyro-oscillation mode $\left(\Omega_{\mathrm{i}}\right)$ if $B_{\mathrm{z} 0}^{\prime}$ is strong enough.

\section{C2.3 Ion inertia neglected}

In this case, $\omega \sim \omega_{\mathrm{e}} \gg \omega_{\mathrm{i}}$. Equation (C23) gives the Electron upper hybrid eUH mode $\left(\omega_{\mathrm{eUH}}\right)$ :

$\omega^{2}=\omega_{\mathrm{e}}^{2}+\omega_{\mathrm{pe}}^{2}=\omega_{\mathrm{eUH}}^{2}(k)$

which leads to the ion gyro-oscillation mode $\left(\Omega_{\mathrm{e}}\right)$ if $B_{\mathrm{z} 0}^{\prime}$ is strong enough. 


\section{C2.4 Extreme cases}

The pseudo-Bohm-Gross frequency $\omega_{\alpha}(k)$ reveals that if the scale of the wavelengths is large (i.e., $k$ is small) compared to the thermal background gyroradius $\rho_{\alpha}=v_{\mathrm{T} \alpha} / \Omega_{\alpha}=\sqrt{m_{\alpha} k_{\mathrm{B}} T_{\alpha}} /\left(e B_{\mathrm{z} 0}^{\prime}\right)$, Debye length, above propagations are only weakly modified by the temperatures of particles. In the extreme case where $k=0$, no propagations exist and particles are only oscillating locally with pure LH frequency, i.e.,

$\omega_{\mathrm{LH}}^{2}=\frac{\omega_{\mathrm{pi}}^{2}}{1+\left(\frac{\omega_{\mathrm{pe}}}{\Omega_{\mathrm{e}}}\right)^{2}}$, or $\frac{\omega_{\mathrm{LH}}}{\omega_{\mathrm{pi}}}=\frac{1}{\sqrt{1+\left(\frac{\omega_{\mathrm{pe}}}{\Omega_{\mathrm{e}}}\right)^{2}}}$

and UH freqiencies, i.e.,

$\omega_{\mathrm{iUH}}^{2}=\Omega_{\mathrm{i}}^{2}+\omega_{\mathrm{pi}}^{2}$, or $\frac{\omega_{\mathrm{iUH}}}{\omega_{\mathrm{pi}}}=\sqrt{\left(\frac{\Omega_{\mathrm{i}}}{\omega_{\mathrm{pi}}}\right)^{2}+1}$

and

$\omega_{\mathrm{eUH}}^{2}=\Omega_{\mathrm{e}}^{2}+\omega_{\mathrm{pe}}^{2}$, or $\frac{\omega_{\mathrm{eUH}}}{\omega_{\mathrm{pi}}}=\sqrt{\left(\frac{\Omega_{\mathrm{e}}}{\omega_{\mathrm{pi}}}\right)^{2}+\left(\frac{\omega_{\mathrm{pe}}}{\omega_{\mathrm{pi}}}\right)^{2}}$

in above respective modes. Notice that this extreme case is equivalent to cold plasma conditions where particle pressures have no effects.

On the contrary, for extreme small-scale wavelengths (i.e., $k$ is large), the dispersion relations of above modes become

$\omega^{2}=\frac{\omega_{\mathrm{pi}}^{2}}{1+\omega_{\mathrm{pe}}^{2} /\left(k v_{\mathrm{T}_{\mathrm{e}}}\right)^{2}}$

$\omega^{2}=\left(\sqrt{\gamma} k v_{\mathrm{T}_{\mathrm{i}}}\right)^{2}+\omega_{\mathrm{pi}}^{2}$

and

$\omega^{2}=\left(\sqrt{\gamma} k v_{\mathrm{T}_{\mathrm{e}}}\right)^{2}+\omega_{\mathrm{pe}}^{2}$

respectively, irrelevant of gyro-frequencies of particles. Under cold plasma conditions, it is easy to see that there exist two non-propagating modes, one of which oscillates in the electron plasma frequency $\omega_{\mathrm{pe}}$, and the other is in the ion plasma frequency $\omega_{\text {pi }}$.

Acknowledgements. This work is funded by Visiting Fellowships in Canadian Government Laboratories Program, Natural Sciences and Engineering Research Council (NSERC) of Canada. The authors thank C. Watt and another anonymous referee for extensive, valuable comments and advices. J. Ma also show gratitudes to J.-P. St.-Maurice for productive guidance and discussions in kinetic and wave studies which triggered this study, and D. J. Knudsen for an effective talk about nonlinear stationary Alfvén waves which facilitated the study.

Edited by: S. Lovejoy

Reviewed by: C. Watt and another anonymous referee

\section{References}

Alexeff, I., Estabrook, K., Hirose, A., Jones, W. D., Neidigh, R. V., Olsen, J. N., Scott, F. R., Stirling, W. L., Widner, M. M., and Wing, W. R.: Understanding Turbulent Ion Heating in the Oak Ridge Mirror Machine, "Burnout V”, Phys. Rev. Lett., 25, 848$851,1970$.

Al-Salti, N. and Shivamoggi, B. K.: Electron-inertia effects on driven magnetic field reconnection, Phys. Plasmas, 10, 42714277, 2003.

Artsimovich, L. A.: Controlled Thermonuclear Reactions, Gordon and Breach, New York, 1964.

Baumjohann, W.: The near-Earth plasma sheet: An AMPTE/IRM perspective, Space Sci. Rev., 64, 141-163, 1993.

Bellan, P. M.: Fundamentals of plasma physics (paperback), Cambridge University Press, 2008.

Berthomier, M., Pottelette, R., Malingre, M., and Khotyaintsev, Y.: Electron-acoustic solitons in an electron-beam plasma system, Phys. Plasmas, 9, 2987-2994, 2000.

Berthomier, M., Pottelette, R., Muschietti, L., Roth, I., and Carlson, C.: Scaling of three dimensional electron phase space density holes observed by FAST in the auroral downward current region, Geophys. Res. Lett., 30, 2148-152, 2003.

Bharuthram, R., Reddy, R. V., Lakhina, G. S., and Singh, N.: Low frequency nonlinear waves in the auroral plasma, Phys. Scripta, T98, 137-140, 2002.

Boyd, T. J. M. and Sanderson J. J.: The physics of plasmas, Cambridge University Press, 2003.

Briand, C.: Plasma waves above the ion cyclotron frequency in the solar wind: a review on observations, Nonlin. Processes Geophys., 16, 319-329, 2009,

http://www.nonlin-processes-geophys.net/16/319/2009/.

Burchill, J. K., Knudsen, D. J., Bock, B. J. J., Pfaff, R. F., Walis, D. D., Clemmons, J. H., Bounds, S. R., and Stenbaek-Nielsen, H.: Core ion interactions with BB ELF, lower hybrid, and Alfvén waves in the high-latitude topside ionosphere, J. Geophys. Res., 109, A01219, doi:10.1029/2003JA010073, 2004.

Cattaert, T. and Verheest, F.: Large amplitude parallel propagating electromagnetic oscillitons, Phys. Plasmas, 12, 012307, doi:10.1063/1.1824038, 2005

Cattell, C., Wygant, J., Dombeck, J., Mozer, F. S., Temerin, M., and Russell, C. T.: Observations of large amplitude parallel electric field wave packets at the plasma sheet boundary, Geophys. Res. Lett., 25, 857-860, 1998.

Cattell, C., Dombeck, J., Wygant, J. R., Hudson, M. K., Mozer, F. S., Temerin, M. A., Peterson, W. K., Kletzing, C. A., Russell, C. T., and Pfaff, R. F.: Comparisons of Polar satellite observations of solitary wave velocities in the plasma sheet boundary and the high altitude cusp to those in the auroral zone, Geophys. Res. Lett., 26, 425-428, 1999.

Chang, T.: Lower-hybrid collapse, caviton turbulence, and charged particle energization in the topside auroral ionosphere and magnetosphere, Phys. Fluids B-Plasma, 5, 2646-2656, 1993.

Chang, T. and Coppi, B.: Lower hybrid acceleration and ion evolution in the suprauroral region, Geophys. Res. Lett., 8, 12531256, 1981.

Chatterjee, P. and Roychoudhury, R.: Effect of finite ion temperature on ion acoustic solitary waves in a two-temperature electronplasma system, Can. J. Phys., 75, 337-343, 1997. 
Chiueh, T. and Diamond, P. H.: Two-point theory of current-driven, ion-cyclotron turbulence, Phys. Fluids, 29, 76-96, 1986.

Craik, A. D. D.: The origins of water wave theory, Annu. Rev. Fluid Mech., 36, 1-28, 2004.

Das, G. C., Sarma, J., Gao, Y.-T., and Uberoi, C.: Dynamical behavior of the soliton formation and propagation in magnetized plasma, Phys. Plasmas, 7, 2374-2380, 2000.

Davidson, R. C.: Methods in nonlinear plasma theory, Academic Press, New York, 1972.

Davydov, A. S.: Solitons in Molecule Systems, McGraw-Hill, 1985.

Delory, G. T.: Rocket observations of VLF waves, electron precipitation and ion heating in the auroral ionosphere, $\mathrm{Ph} . \mathrm{D}$. dissertation, University of California at Berkeley, USA, 1996.

Dovner, P. O., Eriksson, A. I., Boström, R., and Holback, B.: Freja multiprobe observations of electrostatic solitary structures, Geophys. Res. Lett., 21, 1827-1830, 1994.

Drazin, P. G.: Solitons, Cambridge University Press, 1984.

Dubinin, E., Sauer, K., and McKenzie, J. F.: Solitons and oscillitons in cold bi-ion plasmas: A parameter study, J. Plasma Phys., 68, 27-52, 2002.

Dubinin, E., Sauer, K., and McKenzie, J. F.: Nonlinear stationary whistler waves and whistler solitons (oscillitons). Exact solutions, J. Plasma Phys., 69, 305-330, 2003a.

Dubinin, E., Sauer, K., and McKenzie, J. F.: Solitons, oscillitons and stationary waves in a cold $p-\alpha$ plasma, J. Geophys. Res., 108, 1295, doi:10.1029/2002JA009571, 2003b.

Dubinin, E., Sauer, K., and McKenzie, J. F.: Solitons, oscillitons and stationary waves in a warm $p-\alpha$ plasma, J. Geophys. Res., 108, 1296, doi:10.1029/2002JA009572, 2003c.

Dubinin, E. M., Maksimovic, M., Cornilleau-Wehrlin, N., Fontaine, D., Travnicek, P., Mangeney, A., Alexandrova, O., Sauer, K., Fraenz, M., Dandouras, I., Lucek, E., Fazakerley, A., Balogh, A., and Andre, M.: Coherent whistler emissions in the magnetosphere - Cluster observations, Ann. Geophys., 25, 303-315, 2007 , http://www.ann-geophys.net/25/303/2007/.

Dubouloz, N., Treumann, R., Pottelette, R., and Malingre, M.: Turbulence generated by a gas of electron acoustic solitons, J. Geophys. Res., 98, 17415-17422, 1993.

Dupree, T. H.: Theory of phase space density granulation in plasma, Phys. Fluids, 15, 334-344, 1972.

Ergun, R. E., Carlson, C. W., McFadden, J. P., Mozer, F. S., Delory, G. T., Peria, W., Chaston, C. C., Temerin, M., Roth, I., Muschietti, L., Elphic, R., Strangeway, R., Pfaff, R., Cattell, C. A., Klumpar, D., Shelley, E., Peterson, W., Moebius, E., and Kistler, L.: FAST satellite observations of large-amplitude solitary structures, Geophys. Res. Lett., 25, 2041-2044, 1998.

Ergun, R. E.: Magnetic-field-aligned electric fields associated with Debye-scale plasma structures, Plasma Phys. Contr. F., 41, A6173, 1999.

Eriksson, A. I., Holback, B., Dovner, P. O., Boström, R., Holmgren, G., André, M., Eliasson, L., and Kintner, P. M.: Freja observatons of correlated small-scale density depletions and enhanced lower hybrid waves, Geophys. Res. Lett., 21, 1843-1846, 1994.

Gary, S. P.: Theory of space plasma micro-instabilities, Cambridge University Press, 1993.

Gradov, O. M., Stenflo, L., and Sünder, D.: Self-consistent description of solitary surface waves on a plasma cylinder, Phys. Fluids, 27, 597-599, 1984.
Gradov, O. M., Stenflo, L., and Sünder, D.: Solitary surface waves on a magnetized plasma cylinder, J. Plasma Phys., 33, 53-58, 1985.

Hasegawa, A. and Kodama, Y.: Solitons in Optical Communications, Clarendon Press, 1995.

Hirose, A., Alexeff, I., and Jones, W. D.: Dispersion measurements of electrostatic ion waves in a uniform magnetic field, Phys. Fluids, 13, 2039-2044, 1970a.

Hirose, A., Alexeff, I., and Jones, W. D.: Dispersion and damping of electrostatic ion waves in a uniform magnetic field, Phys. Fluids, 13, 1414-1416, 1970b.

Hirose, A., Alexeff, I., and Jones, W. D.: Landau damping of ion acoustic waves in a uniform magnetic field, Phys. Fluids, 13, 1290-1297, 1970c.

Hirose, A. and Alexeff, I.: Electrostatic instabilities driven by currents perpendicular to an external magnetic field, Nucl. Fusion, 12, 315-323, 1972.

Hirose, A.: Drift Instabilities in Magnetically Confined Plasmas: A Brief Overview, Lecture Notes, Autumn College on Plasma Physics International Centre for Theoretical Physics, Trieste, Italy, 2005.

Hirose, A.: Plasma waves (I), Lecture Notes, University of Saskatchewan, 2007.

Høymork, S. H., Pécseli, H. L., Lybekk, B., Trusen, J., and Eriksson, A.: The shape and evolution of lower hybrid density cavities observed by FREJA, 26, 213-217, 2001.

Jones, S. T. and Parker, S. E.: Including electron inertia without advancing electron flow, J. Comput. Phys., 191, 322-327, 2003.

Jovanović, D. and Shukla, P. K.: Nonlinear model for coherent electric-field structures in the magnetosphere, Phys. Rev. Lett., 84, 4373-4376, 2000.

Kakad, A. P., Singh, S. V., Reddy, R. V., Lakhina, G. S., Tagare, S. G., and Verheest, F.: Generation mechanism for electron acoustic solitary waves, Phys. Plasmas, 14, 052305-9, 2007.

Kintner, P. M., Vago, J., Chesney, S., Arnoldy, R. L., Lynch, K. A., Pollock, C. J., and Moore, T. E.: Localized lower hybrid acceleration of ionospheric plasma, Phys. Rev. Lett., 68, 24482451, 1992.

Knudsen, D. J.: Spatial modulation of electron energy and density by nonlinear stationary inertial Alfvn waves, J. Geophys. Res., 101, 10761-10772, 1996.

Knudsen, D. J., Bock, B. J. J., Bounds, S. R., Burchill, J. K., Clemmons, J. H., Curtis, J. D., Eriksson, A. I., Koepke, M. E., Pfaff, R. F., Wallis, D. D., and Whaley, N.: Lower-hybrid cavity density depletions as a result of transverse ion acceleration localized on the gyroradius scale, J. Geophys. Res., 109, A04212, doi:10.1029/2003JA010089, 2003.

Kourakis, I. and Shukla, P. K.: Electron-acoustic plasma waves: oblique modulation and envelope solitons, Phys. Rev. E, 69, p. 036411, 2004.

Kourakis, I. and Shukla, P. K.: Exact theory for localized envelope modulated electrostatic wavepackets in space and dusty plasmas, Nonlin. Processes Geophys., 12, 407-423, 2005, http://www.nonlin-processes-geophys.net/12/407/2005/.

Kuehl, H. H. and Zhang, C. Y.: Effects of ion drift on smallamplitude ion-acoustic solitons, Phys. Fluids B-Plasma, 3, 2628, 1991

LaBelle, J., Kintner, P. M., Yau, A. W., and Whalen, B. A.: Large amplitude wave packets observed in the ionosphere in 
association with transverse ion acceleration, J. Geophys. Res., 91, 7113-7118, 1986.

Lakhina, G. S., Kakad, A. P., Singh, S. V., and Verhest, F.: Ionand electron-acoustic solitons in two temperature space plasmas, Phys. Plasmas, 15, p. 062903, 2008.

Lavraud, B., Borovsky, J. E., Génot, V., Schwartz, S. J., Birn, J., Fazakerley, A. N., Dunlop, M. W., Hasegawa, H., Rouillard, A. P., Berchem, J., Bogdanova, Y., Constantinescu, D., Dandouras, I., Eastwood, J. P., Escoubet, C. P., Frey, H., Jacquey, C., Panov, E., Pu, Z. Y., Shen, C., Shi, J., Sibeck, D. G., Volwerk, M., and Wild, J. A.: Tracing solar wind plasma entry into the magnetosphere using ion-to-electron temperature ratio, Geophys. Res. Lett., 36, L18109, doi:10.1029/2009GL039442, 2009.

Lee, L. C. and Kan, J. R.: Nonlinear ion-acoustic waves and solitons in a magnetized plasma, Phys. Fluids, 24, 430-436, 1981.

Ma, J. Z. G.: Nonlinear ion-acoustic (IA) waves driven in a cylindrically symmetric flow, Astrophys. Space Sci., 0004-640X (Print) 1572-946X (Online), 2010.

Ma, J. Z. G. and Hirose, A.: Parallel propagation of ion solitons in magnetic flux tubes, Phys. Scripta, 79, p. 045502, 2009a.

Ma, J. Z. G. and Hirose, A.: High-frequency electrostatic lowerhybrid (LH) waves in magnetic flux tubes, Phys. Scripta, 79, p. 035503, 2009b.

Ma, J. Z. G., St.-Maurice, J.-P., and Hirose, A.: Non-wave mechanism of transverse ion heating in magnetic flux tubes, Phys. Scripta, 80, p. 025501, 2009.

Mamun, A. A. and Shukla, P. K.: Obliquely propagating electronacoustic solitary waves, Phys. Plasmas, 9, 1474-1477, 2002.

McFadden, J. P., Carlson, C. W., Ergun, R. E., Chaston, C. C., Mozer, F. S., Temerin, M., Klumpar, D. M., Shelley, E. G., Peterson, W. K., Moebius, E., Kistler, L., Elphic, R., Strangeway, R., Cattell, C., and Pfaff, R.: Electron modulation and ion cyclotron waves observed by FAST, Geophys. Res. Lett., 25, 2045-2048, 1998.

McKenzie, J. F., Dubinin, E., Sauer, K., and Doyle, T. B.: The application of the constants of motion to nonlinear stationary waves in complex plasmas: a unified fluid dynamic viewpoint, J. Plasma Phys., 70, 431-462, 2004.

Merlino, R. L. and D'Angelo, N.: Electron and ion inertia effects on current-driven collisional dust acoustic, dust ion acoustic, and ion acoustic instabilities, Plasma Phys., 12, p. 054504, 2005.

Mikhailovskii, A. B.: Theory of plasma instabilities, in: Instabilities of an Inhomogeneous Plasma, vol. 2, Consultants Bureau, New York, 1974.

Nakamura, Y. and Sugai, H.: Experiments on Ion-acoustic Solitons in a Plasma, Chaos Soliton Fract., 7, 1023-1031, 1996.

Pécseli, H. L.: Solitons and weakly nonlinear waves in plasmas, IEEE T. Plasma Sci., 13, 53-86, 1985.

Pécseli, H. L., Iranpour, K., Hotter, Ø., Lybekk, B., Holtet, J., Trulsen, J., Eriksson, A., and Holback, B.: Lower hybrid wave cavities detected by the FREJA satellite, J. Geophys. Res., 101, 5299-5316, 1996.

Phan, T.-D., Paschmann, G., Baumjohann, W., Sckopke, N., and Lühr, H.: The magnetosheath region adjacent to the dayside magnetopause: AMPTE/IRM observations, J. Geophys. Res., 99, 121-141, 1994.

Pickett, J. S., Chen, L.-J., Kahler, S. W., Santolík, O., Goldstein, M. L., Lavraud, B., Décréau, P. M. E., Kessel, R., Lucek, E., Lakhina, G. S., Tsurutani, B. T., Gurnett, D. A., Cornilleau-
Wehrlin, N., Fazakerley, A., Rème, H., and Balogh, A.: On the generation of solitary waves observed by Cluster in the nearEarth magnetosheath, Nonlin. Processes Geophys., 12, 181-193, 2005 , http://www.nonlin-processes-geophys.net/12/181/2005/.

Pinçn, J.-L., Kintner, P. M., Schuck, P. W., and Seyler, C. E.: Observation and analysis of lower hybrid solitary structures as rotating eigenmodes, J. Geophys. Res., 102, 17283-17296, 1997.

Pottelette, R., Ergun, R. E., Treumann, R. A., Berthomier, M., Carlson, C. W., McFadden, J. P., and Roth, I.: Modulated electronacoustic waves in auroral density cavities: FAST observations, Geophys. Res. Lett., 26, 2629-2632, 1999.

Pottelette, R., Treumann, R. A., Berthomier, M., and Jasperse, J.: Electrostatic shock properties inferred from AKR fine structure, Nonlin. Processes Geophys., 10, 87-92, 2003, http://www.nonlin-processes-geophys.net/10/87/2003/.

Pottelette, R. and Berthomier, M.: Nonlinear electron acoustic structures generated on the high-potential side of a double layer, Nonlin. Processes Geophys., 16, 373-380, 2009, http://www.nonlin-processes-geophys.net/16/373/2009/.

Reddy, R. V., Lakhina, G. S., Singh, N., and Bharuthram, R.: Spiky parallel electrostatic ion cyclotron and ion acoustic waves, Nonlin. Processes Geophys., 9, 25-29, 2002,

http://www.nonlin-processes-geophys.net/9/25/2002/.

Retterer, J., Chang, T., and Jasperse, J.: Ion acceleration by lower hybrid waves in the suprauroral region, J. Geophys. Res., 91, 1609-1618, 1986.

Sauer, K., Dubinin, E., and McKenzie, J. F.: New type of soliton in Bi-ion plasmas and possible implications, Geophys. Res. Lett., 28, 3589-3592, 2001.

Sauer, K., Dubinin, E., and McKenzie, J. F.: Wave emission by whistler oscillitons: Application to "coherent lion roars", Geophys. Res. Lett., 29(24), 2226, doi:10.1029/2002GL015771.

Sauer, K., Dubinin, E., and McKenzie, J. F.: Solitons and oscillitons in multi-ion space plasmas, Nonlin. Processes Geophys., 10, 121-130, 2003, http://www.nonlin-processes-geophys.net/10/121/2003/.

Schuck, P. W., Bonnell, J. W., and Kintner Jr., P. M.: A review of lower hybrid solitary structures, IEEE T. Plasma Sci., 31, 11251177, 2003.

Sen, B., Das, B., and Chatterjee, P.: Effect of electron inertia on large amplitude solitary waves in presence of kinematic viscosity in dusty plasma, Eur. Phys. J. D, 49, 211-216, 2008.

Shapiro, V. D.: Modulational interaction of the Lower-hybrid waves with a kinetic-Alfvén mode, Phys. Rev. Lett., 81, 3415-3418, 1998.

Shi, J. K., Xu, B. Y., Torkar, K., and Liu, Z. X.: Nonlinear waves in a low- $\beta$ plasma with cylindrical symmetry, Phys. Plasmas, 8 , p. 4780, 2001.

Shi, J. K., Zhang, T., Torkar, K., and Liu, Z. X.: An interpretation of electrostatic density shocks in space plasma, Phys. Plasmas, 12, p. 082901, 2005.

Shi, J., Qureshi, M. N. S., Torkar, K., Dunlop, M., Zhenxing Liu, and Zhang, T. L.: An interpretation for the bipolar electric field structures parallel to the magnetic field observed in the auroral ionosphere, Ann. Geophys., 26, 1431-1437, 2008, http://www.ann-geophys.net/26/1431/2008/.

Shivamoggi, B. K.: Plasma dynamics with electron-inertia effects, J. Plasma Phys., 58, 329-344, 1997. 
Shukla, P. K. and Yu, M. Y.: Exact solitary ion acoustic waves in a magnetoplasma, J. Math. Phys., 19, 2506-2508, 1978.

Shukla, P. K., Mamun, A., and Eliasson, B.: 3-D electron-acoustic solitary waves introduced by phase space electron vortices in magnetized space plasmas, Geophys. Res. Lett., 31, L07803, doi:10.1029/2004GL019533, 2004.

Singh, S. V. and Lakhina, G. S.: Electron acoustic solitary waves with non-thermal distribution of electrons, Nonlin. Processes Geophys., 11, 275-279, 2004,

http://www.nonlin-processes-geophys.net/11/275/2004/.

Stenflo, L.: Strongly nonlinear cylindrical surface waves, Phys. Scripta, 41, 643-644, 1990.

Stenflo, L. and Yu, M. Y.: Nonlinear waves in a rotating plasma cylinder, IEEE T. Plasma Sci., 20, 104-105, 1992.

Stenflo, L. and Yu, M. Y.: Nonlinear waves in cylindrically bounded magnetized plasmas, Phys. Rev. E, 51, 1408-1411, 1995.

Sydora, R. D., Sauer, K., and Silin, I.: Coherent whistler waves and oscilliton formation: Kinetic simulations, Geophy. Res. Lett., 34, L22105, doi:10.1029/2007GL031839, 2007.

Tanaca, H., Koganei, M., and Hirose, A.: Drift instability in mercury-vapor discharges, Phys. Lett., 23, 679-680, 1966a.

Tanaca, H., Koganei, M., and Hirose, A.: Dispersion relation of ion waves in Mercury-vapour discharges, Phys. Rev. Lett., 16, 1079-1081, 1966b.

Tanaca, H., Hirose, A., and Koganei, M.: Ion wave instabilities in Mercury-vapor plasma, Phys. Rev., 161, 94-101, 1967.

Temerin, M., Woldorff, M., and Mozer, F. S.: Nonlinear Steepening of the Electrostatic Ion Cyclotron Wave, Phys. Rev. Lett., 43, 1941-1943, 1979.

Tjulin, A., Eriksson, A. I., and André, M.: Lower hybrid cavities in the inner magnetosphere, Geophys. Res. Lett., 30, p. 1364, 2003.
Tjulin, A., André, M., Eriksson, A. I., and Maksimovic, M.: Observations of lower hybrid cavities in the inner magnetosphere by the Cluster and Viking satellites, Ann. Geophys., 22, 29612972, 2004, http://www.ann-geophys.net/22/2961/2004/.

Tsurutani, B. T., Galvan, C., Arballo, J. K., Winterhalter, D., Sakurai, R., Smith, E. J., Buti, B., Lakhina, G. S., and Balogh, A.: Relationship between discontinuities, magnetic holes, magnetic decreases, and nonlinear Alfvén waves: Ulysses observations over the solar poles, Geophys. Res. Lett., 29(11), 1528, doi:10.1029/2001GL013623, 2002a.

Tsurutani, B. T., Dasgupta, B., Galvan, C., Neugebauer, M., Lakhina, G. S., Arballo, J. K., Winterhalter, D., Goldstein, B. E., and Buti, B.: Phase-steepened Alfvén waves, proton perpendicular energization and the creation of magnetic holes and magnetic decreases: The ponderomotive force, Geophys. Res. Lett., 29, 24pp., $2002 b$.

Tsurutani, B. T., Lakhina, G. S., Pickett, J. S., Guarnieri, F. L., Lin, N., and Goldstein, B. E.: Nonlinear Alfvén waves, discontinuities, proton perpendicular acceleration, and magnetic holes/decreases in interplanetary space and the magnetosphere: intermediate shocks?, Nonlin. Processes Geophys., 12, 321-336, 2005, http://www.nonlin-processes-geophys.net/12/321/2005/.

Uberoi, C.: Electron-Inertia Effects On The Transverse Gravitational Instability, J. Plasma Fusion Res. SERIES, 8, 823-825, 2009.

Vladimirov, S. V., Yu, M. Y., and Stenflo, L.: Surface Wave Solitons in an Electronic Medium, Phys. Lett. A, 174, 313-316, 1993.

Yu, M. Y., Shukla, P. K., and Bujarbarua, S.: Fully nonlinear ionacoustic solitary waves in a magnetized plasma, Phys. Fluids, 23, 2146-2147, 1980.

Yu, M. Y. and Stenflo, L.: Nonlinear waves in a plasma cylinder, IEEE T. Plasma Sci., 19, 641-644, 1991. 